\title{
Smart Cardiac Framework for an Early Detection of Cardiac Arrest Condition and Risk
}

\author{
Apeksha Shah ${ }^{1}$, Swati Ahirrao ${ }^{1}$, Sharnil Pandya ${ }^{1 *}$, Ketan Kotecha $^{2}$ and Suresh Rathod ${ }^{1}$ \\ ${ }^{1}$ Computer Science Department, Symbiosis Institute of Technology, Symbiosis International (Deemed University), Pune, \\ India, ${ }^{2}$ Symbiosis Centre for Applied Artificial Intelligence, Symbiosis Institute of Technology, Symbiosis International (Deemed \\ University), Pune, India
}

\section{OPEN ACCESS}

Edited by:

Thippa Reddy Gadekallu, VIT University, India

Reviewed by:

Chintan Modi,

Independent Researcher,

Sarnia, Canada

Praveen Kumar,

VIT University, India

Abdul Rehman Javed,

Air University, Pakistan

*Correspondence:

Sharnil Pandya

sharnil.pandya@sitpune.edu.in

Specialty section:

This article was submitted to

Digital Public Health,

a section of the journal

Frontiers in Public Health

Received: 21 August 2021 Accepted: 20 September 2021

Published: 22 October 2021

Citation:

Shah A, Ahirrao S, Pandya S, Kotecha K and Rathod S (2021)

Smart Cardiac Framework for an Early Detection of Cardiac Arrest Condition and Risk.

Front. Public Health 9:762303. doi: 10.3389/fpubh.2021.762303
Cardiovascular disease (CVD) is considered to be one of the most epidemic diseases in the world today. Predicting CVDs, such as cardiac arrest, is a difficult task in the area of healthcare. The healthcare industry has a vast collection of datasets for analysis and prediction purposes. Somehow, the predictions made on these publicly available datasets may be erroneous. To make the prediction accurate, real-time data need to be collected. This study collected real-time data using sensors and stored it on a cloud computing platform, such as Google Firebase. The acquired data is then classified using six machine-learning algorithms: Artificial Neural Network (ANN), Random Forest Classifier (RFC), Gradient Boost Extreme Gradient Boosting (XGBoost) classifier, Support Vector Machine (SVM), Naïve Bayes (NB), and Decision Tree (DT). Furthermore, we have presented two novel gender-based risk classification and age-wise risk classification approach in the undertaken study. The presented approaches have used Kaplan-Meier and Cox regression survival analysis methodologies for risk detection and classification. The presented approaches also assist health experts in identifying the risk probability risk and the 10-year risk score prediction. The proposed system is an economical alternative to the existing system due to its low cost. The outcome obtained shows an enhanced level of performance with an overall accuracy of $98 \%$ using DT on our collected dataset for cardiac risk prediction. We also introduced two risk classification models for gender- and age-wise people to detect their survival probability. The outcome of the proposed model shows accurate probability in both classes.

Keywords: artificial intelligence, cardiac arrest prediction, machine learning, predictive analysis, risk classification, heart failure

\section{INTRODUCTION}

At present times, cardiovascular disease (CVD) is one of the most contagious illnesses. According to WHO, $\sim 60 \%$ of cardiac patients are Indians to suffer from CVDs. Cardiac symptoms are generally associated with dynamic changes in an individual. The vital changes in the human body may lead to cardiac problems due to misdiagnoses or improper treatment. Another sign of cardiac disease is environmental changes and lifestyles of people (1). In healthcare, CVDs are considered a vital aspect to be diagnosed as soon as possible to minimize the risk. Hence, early prediction of CVDs needs to be examined in a patient $(2,3)$. 
Cardiovascular disease comprises heart or cardiac diseases in heart patients and is a critical challenge in the medical field. Several reasons and findings that cause cardiac arrest are observed, such as a change in personal and professional lifestyles, habits, inactive lifestyle, growing age, habitual history related to smoking, alcohol consumption, stress level, and physiological signs, such as diabetes, high level of blood pressure (BP), obesity, cholesterol, hypertension, and existing heart problems. These risk factors need early, accurate, and efficient diagnosis to prevent cardiac arrests (4-6). The risk factors can be depreciated by executing proper lifestyle activities, such as lowering salt usage, absorbing a healthy diet, prohibiting alcohol and tobacco use, and regular physical exercise (7). Medical sectors use the abovementioned vital signs to produce consequential information from data. The vital signs are generally collected from publicly available datasets, wearable gadgets, medical hospitals, or sensors. Monitoring health parameters using Internet-of-Things (IoT) is a forming trend for future well-being. IoT sensors are mostly used to collect real-time vital signs and monitor the health parameters of individuals (8). Collecting, processing, and analyzing vitals help predict risk early to tackle the problem $(9,10)$.

One of the broad areas of Artificial Intelligence (AI) is machine learning (ML). ML is an effective technology and efficient field, which is based on prediction purposes. Using ML concepts, we can develop models or enable human abilities and train our data collected or gathered from the past for future predictive analysis (11). Those data are further divided into training and testing, which help in predicting future possibilities. This combined technology is called ML (12). The ML algorithm needs pertinent information for training and testing. In addition, the model performance can be raised if the balanced data are given to the ML model. In addition to that, the capability and accuracy of the predictive model can be improved if relevant features are selected from a dataset. Hence, a balanced dataset and feature selection are paramount for improving model performance (13). Furthermore, for cardiac arrest prediction, the $\mathrm{ML}$ algorithm is used, which has been addressed in this research. Nowadays, deep learning models show prominent growth and improvement in predicting and analyzing heart diseases (14).

Several studies have shown predictions on publicly available datasets. Still, this research aims to find early risk prediction of cardiac arrest based on real-time data collected from an individual using sensors and equipment. Furthermore, the authors build a Neural Network, Bagging, and Boosting model using ML techniques by selecting relevant datasets to show which model accomplishes best. This study also aims to classify risk in different age and gender groups of people. The points mentioned below show the main contributions of the article:

- To collect real-time vital signs of an individual using sensors and equipment.

- To detect cardiac arrest by applying the ML algorithm.

- To show a comparative analysis of the various ML classifiers.

- To classify cardiac arrest risk for the coming 10 years in different age groups.
- To show survival probability gender-wise in our creation of data.

The rest of the article is assembled as follows. Section Related Work narrates research work on heart diseases along with existing methods, datasets, and techniques available. Section Proposed Work exhibits the study of the proposed study of the author. Section Methodology reveals the methodologies and algorithms used for disease risk prediction. Section Risk Classification conveys the risk classification in people. Section Exploratory Data Analysis presents the results of Exploratory Data Analysis. Section Implementation Details communicates the implementation details of all the experiments and results. Finally, section Discussion, Conclusion, and Future Work discusses and concludes the research study of the author.

\section{RELATED STUDY}

\section{Literature Survey}

This section discusses the existing article to predict and diagnose heart diseases using several techniques and datasets used with various features and classification techniques.

Ambekar and Phalnikar (1) proposed a risk model for heart disease with structured data using a Convolutional Neural Network-based Unimodal Disease Risk Prediction (CNNUDRP) model to classify risk levels as high, low, and medium. The dataset was extracted from the UCI repository. To avoid missing values, performed data cleaning and imputation to substitute missing values and increase the performance of the model were implemented. The proposed model achieves an accuracy of nearly about 65\%. Ramprakash et al. (2) developed a model using a Deep Neural Network (DNN) and $\chi^{2}-\mathrm{a}$ statistical method to predict the risk of heart patients. The Cleveland dataset was used, which is publicly available on the UCI repository. In this study, DNN models Artificial Neural Network (ANN) and DNN and proposed statistical methods $\chi^{2}$ ANN and $\chi^{2}$-DNN were compared. The comparison shows that the proposed method $\chi^{2}$-DNN was more efficient in providing an accurate accuracy of $94 \%$. Maini et al. (5) discuss data mining techniques to detect heart disease risk at an early stage and show the importance of assigning a cloud-based approach for helping healthcare providers. Mohan et al. (6) proposed a hybrid model combination of Linear model and Random Forest (RF) technique with different features combined on publicly available datasets, i.e., Cleveland from UCI repository and compared its result with models of Naïve Bayes (NB), RF, Linear model, Deep Learning, Logistic regression, etc. From all the proposed models, Random Forest with Linear Model (HRFLM) outperforms all others with $88.4 \%$ accuracy. Shah et al. (3) used the Cleveland dataset available from the UCI repository. They implemented ML algorithms, such as Decision Tree (DT), K-Nearest Neighbor (KNN), NB, and RF for visualizing the probability of heart disease in the patients. Among all models, KNN shows the highest accuracy score of $90 \%$ in predicting heart disease.

Garg et al. (15) used an ML algorithm to predict and classify diseases of a person who is suffering from heart problems. They have used a dataset from Kaggle, which was commonly used 
for prediction purposes. From the attributes, 13 main attributes, such as the age of a person, chest pain, cholesterol level, and many more, were considered to predict heart diseases. The ML algorithms used are KNN and RF. The comparison was made between the ML algorithm and RF achieves the highest accuracy with $82 \%$. Yadav et al. (16) proposed an optimization model, namely, "Optimized DNN using Talos" deploy ML classifiers, such as DT, KNN, RF, and Ensemble model (used ANN, KNN, and Support Vector Machine, SVM), for the prediction of heart diseases. They have used the concept of dimensional reduction where only vital information of patients was considered. On comparing their model with traditional models, the proposed model provides better accuracy and prediction.

Latha and Jeeva (17) convey classification as a commonly used technique in ML algorithms, so they have tried a new technique that is an ensemble technique that combines two or more classifiers to improve the accuracy obtained by a simple classification technique. They focus on increasing the accuracy and implementation of feature selection with a different set of features. There was a significant increment in the prediction accuracy. Singh et al. (18) proposed a multisurface proximal SVM (MPSVM)-based DT, collaborated with the ensembling method consisting of Gradient Boosting and RF algorithms on Cleveland dataset from UCI repository. They have solved the 2-class problem and the 5-class problem on a dataset using the proposed method. On analyzing, the 2class problem shows better accuracy of $91 \%$ than the 5-class problem with $73 \%$. Sowmiya and Sumitra (19) used the Cleveland dataset from the UCI repository to implement heart disease prediction. They have applied an ant colony optimization technique for the best feature selection for the hybrid KNN classifier. This hybrid model is compared to other classification algorithms, such as SVM, KNN, NB, C4.5, and DT. Their study proposed $\mathrm{HKNN}$ as an effective and efficient technique for heart disease prediction.

Alotaibi (20) focused on improving the performance and accuracy of ML algorithms compared with the previous studies. In this study, the prediction of heart diseases is made using RapidMiner tool on Cleveland dataset extracted from UCI repository and compared the results with previous work that used Weka and MATLAB tools where the performance of RapidMiner tool gives higher accuracy using techniques: SVM, DT, and Logistic regression.

Nikookar and Naderi (21) proposed a hybrid ensemble method to show comparison with the primary ensemble method using SPECT heart disease dataset, consists of SPECT images based on feature vector on fuser classifier algorithm, i.e., Adaboost, MLP, Logitboost, and RF. The comparison was made between hybrid and basic ensemble classifiers where the proposed hybrid classifier shows higher accuracy. Tama et al. (22) proposed a model using a two-tier ensemble technique to detect heart disease. The developed model was implemented by ensembling gradient boosting, RF, and extreme gradient boosting (XGBoost) classifiers. The model was trained and validated on available datasets: Cleveland, Statlog, Hungarian, and ZAlizadeh Sani. A two-step significance test was conducted to compare with the existing article and provided the highest results. Li et al. (13) developed a diagnosis system called Fast Conditional Mutual Information (FCMIM-SVM) based on classification algorithms-ANN, SVM, and Logistic Regressionto diagnose heart diseases using the Cleveland dataset. They used minimal redundancy maximal relevance, relief, most minor absolute shrinkage selector operator, etc., as a feature selection algorithm to select relevant features to get accurate accuracy. The proposed model shows a better accuracy as compared to the previously developed model. Sarmah (8) proposed a model using IOT sensors attached to the body of the patient to gather real-time data and applied the Deep learning modified neural network (DLMNN) model for heart disease prediction. The prediction from the model executes in three ways: Authentication, Encryption, and Classification. Thus, the classification shows normal and abnormal output and prescribes it in its manner. Hence, the model proposed shows improvement over the existing algorithm with providing authentication.

Chauhan et al. (10) used an ML algorithm to predict heart diseases on the Cleveland dataset publicly available and compared the result with the algorithms. Among all, ANN outperforms all classification algorithms with $85 \%$ accuracy. Pan et al. (14) proposed an enhanced deep-learning Convolution Neural Network (CNN) model that predicts and improves heart disease in a patient. This model uses the more profound architecture of the multilayer perceptron model and regularizes parameters. In addition, this system has been executed on the Internet-of-Medical-Things (IOMT) platform to provide efficient solutions for a doctor for the diagnosis of heart diseases. This CNN model is compared with other ML techniques and shows a maximum accuracy of $97 \%$. Fitriyani et al. (7) developed a clinical decision support system (CDSS) to recognize early heart failure. They also proposed the Heart Disease Prediction Model (HDPM) for the CDSS system to eliminate noise and outlier presence. They have built the Starlog and Cleveland dataset model, which is available publicly and tested on other ML models and compared the results. The proposed model achieves 95\% with Statlog and 98\% with the Cleveland dataset.

Tate and Rao (23) give an idea of using wearables in form of a wristwatch, wrist band, ambulatory devices, skin response monitors, Fitbit tracker with an app installed, and many more for prediction of cardiac arrest at an early stage. These electronic gadgets help in tracking our day-to-day routine and monitoring our daily activities by keeping track of each activity. The vital information of patients can assist individuals by sending emergency alerts via web and mobile interfaces. Several studies, such as Framingham Heart Study, had developed the Cox regression hazard ratio model specifically for the sex groups for the prediction of heart diseases by estimating 10-year risk. Hence, the idea of capturing the vitals of an individual from wearables helps to get a better prediction and classification rates for detecting heart diseases. Prabhu et al. (24) proposed the CNBased Multimodal Disease Risk Prediction (CNN-MDRP) model for risk prediction of diseases on a sizeable Medical dataset and compared the result with the algorithm that is CNN-UDRP. The performance of CNN-MDRP shows more improvement than the CNN-UDRP model for risk prediction. Shankar et al. (25) aim to predict heart diseases in an individual. They have made the 
prediction based on details entered by a patient in a hospital, and the data were trained on a CNN model to find the accuracy. The data used were in structured and unstructured formats. The proposed CNN model shows improvement by comparing it with other algorithms.

Singh and Kumar (12) have calculated accuracy for predicting heart diseases using ML algorithms. The algorithms were KNN, DT, Linear Regression, and SVM utilizing Cleveland dataset from the UCI repository for training and testing. Overall, the accuracy of the KNN algorithm achieves $87 \%$ for early heart disease prediction. Amin et al. (26) have collected real-time data, i.e., the vital signs of a patient using Fitbit wearable. The data captured by the wearable were entered into an application and processed through an ML algorithm. This study aims to notify an individual whether he/she is at risk of CVD or not. The outcome of the prediction result helps doctors in diagnosis patient health. Chen et al. (27) proposed a technique for predicting the occurrence of perioperative heart diseases. The data were collected from the hospital and divided into structured data in numerical form and unstructured data in textual form. ML algorithms for numerical data gradient boosting tree algorithm and textual data topic model of text-based data model were used. The datasets were fused using a simple logistic regression model and achieved a sensitivity of $90 \%$ and specificity of $93 \%$.

Cohen et al. (28) proposed a 1-year risk prediction model for patients with congenital heart diseases. The dataset was collected from Quebec hospitals of patients varying in age between 18 and 64 years. Multivariate logistic regression was used to predict the risk for heart diseases. The risk score was indicated between 0 and 19. Hence, the risk score model shows excellent performance. Chang et al. (29) use an ML classification algorithm to predict the vital signs of a patient for the next hour. Vital signs, such as heart rate (HR), BP, mean arterial pressure, and oxygen, were considered. Early warning signs can help nurses and doctors treat the patient before the situation degrades. ML algorithms, such as ANN, RF, Gradient Boosting, and long-short-term-memory (LSTM), were developed. The outcome shows the accuracy of ML models. Youssef Ali Amer et al. (30) have carried our experiment with vital signs of patients using wearable devices. The vital signs considered were BP, oxygen level, and HR. These vital signs were estimated every minute using mean and minimum statistical values.

Then, the ML technique of KNN LS SVM was executed to predict future values of vital signs that were monitored. The performance of implemented ML algorithm was compared with the LSTM approach, and the proposed approach shows much improvement in predicting the early warning score of vital signs. Stehlik et al. (31) have indicated re-hospitalization of HF by non-invasive and remote monitoring. Wearable sensors were placed on the chest of a person (up to 3 months), collecting and recording physiological data (vital signs). The data were uploaded on a cloud platform via a smartphone. Analytics were performed on data to detect HF exacerbation. The result shows $76-88 \%$ sensitivity and $85 \%$ specificity. An alert was shown 6 days prior for re-admission.

Jia et al. (32) use the Cox proportional hazards regression model to find the probability of risk for 10 years using the
Framingham dataset. The risk factors considered were sex, body mass index (BMI), systolic BP (SBP), and diabetes. They have calculated a sex-based risk score using the Cox regression hazard equation.

Yang et al. (33) proposed a multivariate regression model for the prediction of CVDs. The prediction was made on the collection of vital information of patients from the centers of the national high-risk program by having a cardiac events assessment. The dataset consists of all information, such as $\mathrm{BP}$, cholesterol level, obesity, smoking, BMI, and many more attributes were taken into consideration. The area under curve results of the multivariate regression model were then compared to other ML classification techniques, such as CART, NB, and Ada Boost. The outcome result of the multivariate regression model outperforms other methods. Made et al. (34) surveyed CVDs estimating 10-year risk in the different age groups of people. They proposed a SCORE model that uses risk factors, such as weight, $\mathrm{BP}$, cholesterol level, smoking habit, diabetes, and many more. The data were collected by taking samples of men and women of age 18 and above. The 10-year risk estimation was made on statistical analysis, which estimates coronary heart diseases and no-coronary heart disease. These studies on 10 -year risk scores help to prevent heart diseases in the coming future.

\section{Comparison of Previous Findings and Proposed Study}

The literature study discussed above and in Table 1 shows the importance of the vital signs of a person in the healthcare industry to detect health-related problems. Mainly, health problems are majorly concerned with the heart, such as cardiac arrest, CVDs, coronary heart disease, and many more. In this research, the authors have depicted the detection of cardiac arrest in a person by collecting real-time data. Moreover, most of the detection and prediction of heart disease is majorly made on a public dataset consisting of shared attributes. In our contribution, the features chosen are mainly focused on cardiac arrest detection. After detecting cardiac arrest, genderwise survival probability, and age-wise cardiac arrest risk scores are also formulated using two approaches: (1) Kaplan-Meier and (2) Cox regression model. These two methods help to provide which gender has fewer survival chances and which age group has a high probability of risk in the next 10 years.

\section{PROPOSED WORK}

This section briefly describes the novelty of the proposed work (contributions) and the articles mentioned early by some research on the same Cox regression model.

The progress in CVD risk models is ordinarily based on conventional laboratory-based predictors. The primary purpose was to develop a prediction model, which was based on a 10year risk. To predict 10-year risk, a Cox regression proportional method was applied. A dataset from Framingham Original Cohort and SCORE of men and women aged between 30 and 62 was taken to predicate CVD risk. In this study, the CVD risk was 
TABLE 1 | Comparison of literature work and proposed work.

\begin{tabular}{|c|c|c|c|}
\hline References & Findings & Method used & Results obtained \\
\hline Ramprakash et al. (2) & $\begin{array}{l}\text { Prediction of heart disease on Cleveland } \\
\text { dataset }\end{array}$ & $\begin{array}{l}\text { Machine learning algorithms-Deep Neural } \\
\text { Network and } \chi^{2}-\text { a statistical method }\end{array}$ & Accuracy-94\% \\
\hline Mohan et al. (6) & $\begin{array}{l}\text { Prediction of heart disease on Cleveland } \\
\text { dataset }\end{array}$ & $\begin{array}{l}\text { Proposed HRFLM model using Random } \\
\text { Forest, and Linear model }\end{array}$ & Proposed model accuracy - 88\% \\
\hline Sowmiya and Sumitra (19) & $\begin{array}{l}\text { Used Cleveland dataset to implement heart } \\
\text { disease prediction }\end{array}$ & $\begin{array}{l}\text { Used ant colony optimization technique for } \\
\text { hybrid KNN classifier and compared with other } \\
\text { ML models }\end{array}$ & $\begin{array}{l}\text { Proposed approach HKNN proves } \\
\text { efficient and effective technique }\end{array}$ \\
\hline Made et al. (34) & Estimation of 10-year coronary heart risk & $\begin{array}{l}\text { Using statistical approach-regression analysis } \\
\text { in age group of } 18 \text { and above }\end{array}$ & $\begin{array}{l}\text { Study estimates heart risk for the next } \\
10 \text {-years }\end{array}$ \\
\hline Proposed work & $\begin{array}{l}\text { Detection and classification of cardiovascular } \\
\text { diseases in different age-based group and } \\
\text { gender-based people, collected vital signs of } \\
\text { an individual using wearables and medical } \\
\text { equipments }\end{array}$ & $\begin{array}{l}\text { Used } 6 \text { Machine Learning algorithm-Decision } \\
\text { Tree, SVM, ANN, RFC, XGBoost, and NB. } \\
\text { Further, comparison is made among } 6 \\
\text { classifiers. Also, we developed two models } \\
\text { based on Kaplan-Meier method (For } \\
\text { Gender-Based) and Cox-Regression } \\
\text { Proportional Model (For Age-Based) using } \\
\text { scoresheet }\end{array}$ & $\begin{array}{l}\text { Among } 6 \text { Machine learning models- } \\
\text { Decision Tree achieves maximum } \\
\text { accuracy of } 98 \% \text {. Our proposed } \\
\text { approach, for age-based and } \\
\text { gender-based shows risk score (in } \\
\text { percentage) of cardiac arrest for the } \\
\text { next 10-years }\end{array}$ \\
\hline
\end{tabular}

being predicted on a gender basis (men/woman). The risk factors involved are age, sex, BMI, SBP, and diabetes.

A proposed study related to cardiac arrest prediction using the real-time dataset is classified based on gender and age. Our study has used two methods to find the risk classification probabilities among gender-based and age-based. The two methods usedKaplan-Meier survival analysis and Cox regression model survival analysis-are considered. These two methods have their importance in analyzing risk probabilities.

\section{Kaplan-Meier Method}

This method is a non-parametric survival function for the estimation of survival probability. Kaplan-Meier method also estimates the survival curve by giving a statistical comparison between two groups: men and women.

\section{Cox Regression Proportional Model}

Cox regression proportional model does regression analysis with survival data without making any strong assumption. It calculates the hazard ratio (HR) of the covariates used in our data and is based on the equation, and it shows risk probability. Cox regression proportional model is a model that helps to find risk probability or score on more than one covariate. In general, it is a method to identify the effect of variables at some time interval on some event that occurs.

\section{METHODOLOGY}

\section{Measuring Device}

The risk factors associated with heart diseases are cardiac arrest prediction: age, gender, height, weight, BMI, BP [SBP and diastolic BP (DBP)], HR (beats per minute), and oxygen level $\left(\mathrm{SPO}_{2} \%\right)$.

The above factors used in this research are collected using IOT sensors and equipment for different risk factors. The measuring device can capture the vital signs of a person needed to estimate
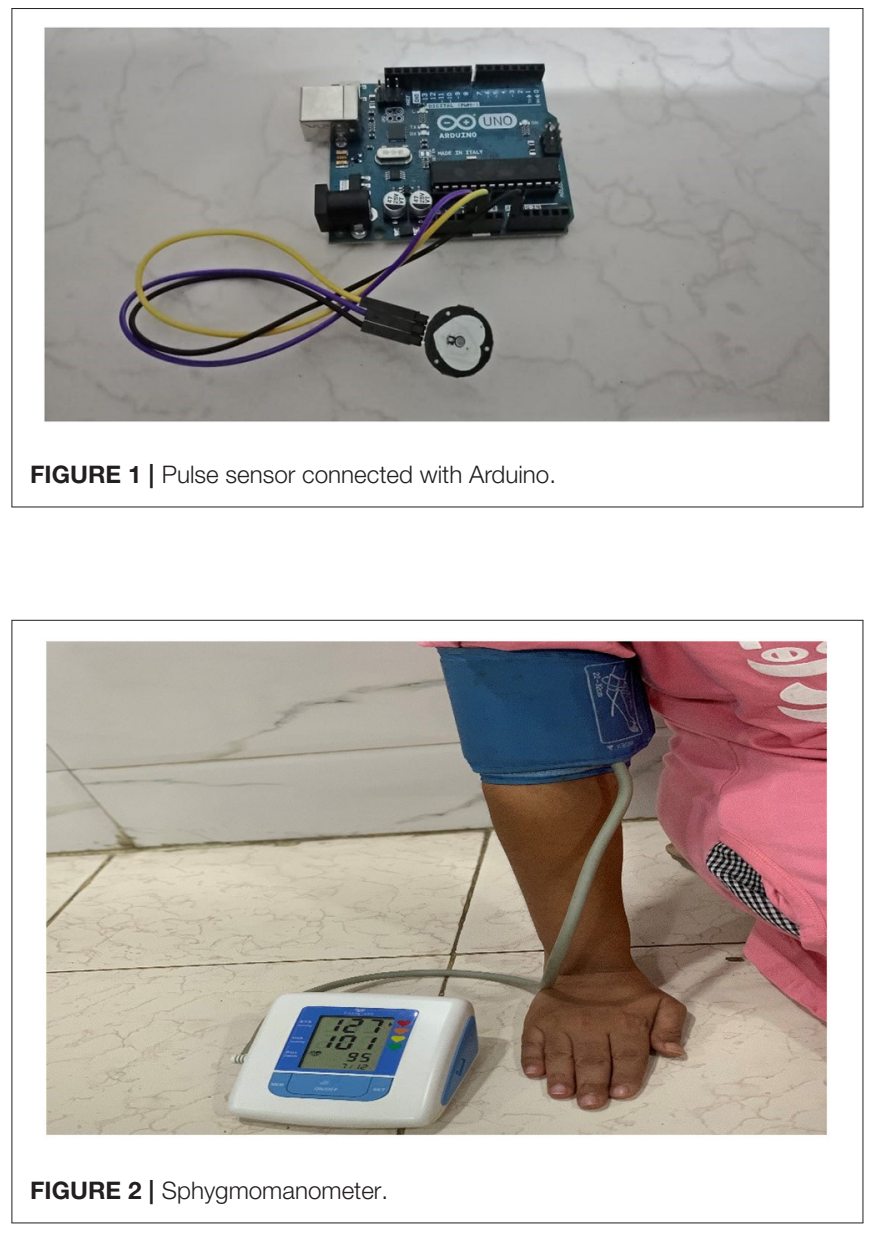

cardiac arrest prediction. Figures 1-3 show measuring device used for capturing vital signs. 


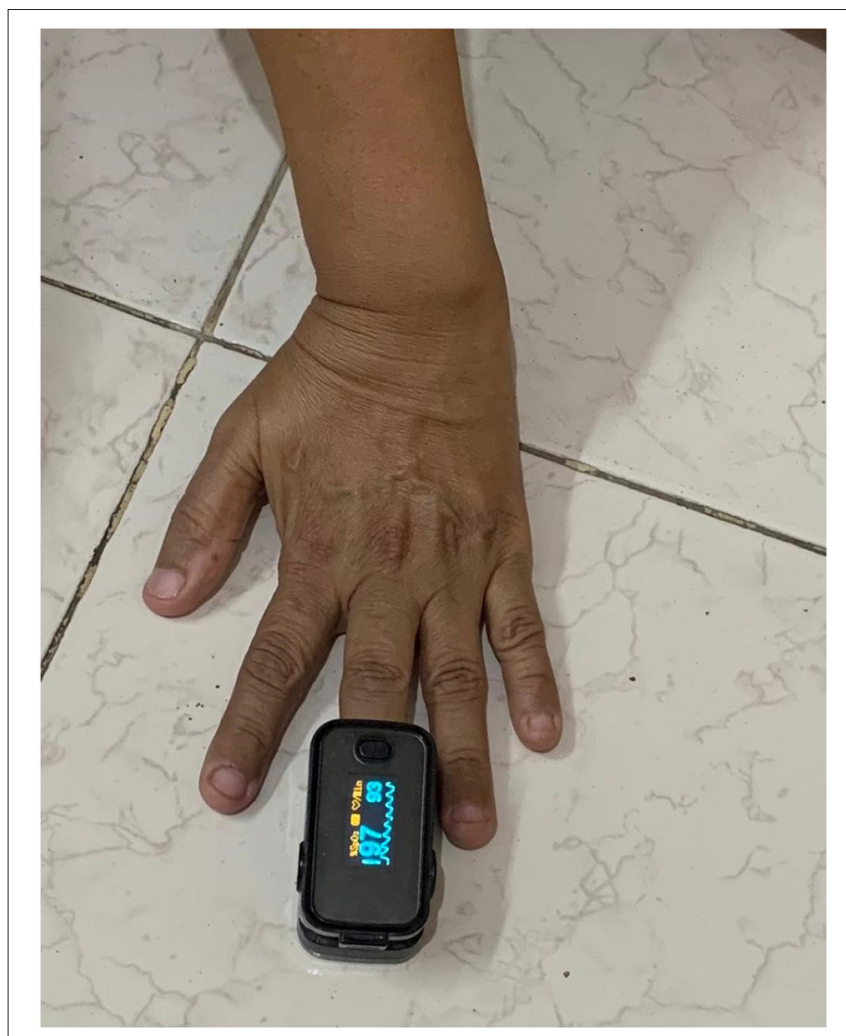

FIGURE 3 | Oximeter.

\section{Data Collection}

\section{Pulse Sensor}

A pulse sensor is a device that is used to detect biometric pulse rate or $\mathrm{HR}$, and it also keeps track of volume change. It is generally helpful for healthcare applications to capture the pulse rate of an individual. A pulse rate is detected when there is a change in blood vessel volume, and that occurs when the heart pumps blood.

For this research, we used a pulse sensor that is connected to Arduino UNO Board. Above, Figure 1 shows the configuration of the pulse sensor and Arduino Board. A pulse rate or HR was captured from a total of 18 patients in beats per minute (BPM) at a 2 min interval.

\section{Sphygmomanometer}

A sphygmomanometer is a device used to measure the BP of a person. The connected rubber cuff with the device is wrapped around the upper arm of a patient and records the blood pressure in SBP and DBP.

For this research, we used Sphygmomanometer to measure BP. Above, Figure 2 shows a Sphygmomanometer connected to the arm of a patient. BP was captured from the same 18 patients at every 2 min interval.

\section{Pulse Oximeter}

The pulse oximeter is a measuring device used to keep track of oxygen levels in a human body. This is an easy and painless measuring device to see how much oxygen reaches the different parts of the body. This device is generally used in hospitals and at home to determine the oxygen level in the body. It measures the oxygen level in $\mathrm{SPO}_{2} \%$ form.

For this research, we used a pulse oximeter to measure the $\mathrm{SPO}_{2}$. Above, Figure 3 shows a pulse oximeter placed on the fingertip of a patient and the estimated oxygen level. It is a noninvasive method for monitoring oxygen saturation. Oxygen level was captured from the same 18 patients at every 2 min interval.

\section{Dataset Description}

Table 2 shows the names of attributes along with their feature description. The table also contains a range column indicating the range value mentioned in our dataset and its dataset. The dataset comprised attributes are:

- Date and time

- Gender

- Age

- Height

- Weight

- BMI

- SBP

- DBP

- $\operatorname{HR}(\mathrm{BPM})$

- Cardiac arrest prediction-target variable.

Cardiac arrest prediction is our target variable from the features mentioned above, which is predicted in our dataset.

The target variable-cardiac arrest prediction-is labeled using the following conditions, as mentioned in Table 3.

\section{Data Pre-processing}

In the ML process, the processing of datasets is essential for good impersonation. Some techniques for data pre-processing are data cleaning, data integration, data transformation, data reduction, and data discretization. In our dataset, there are 540 records where the column gender is denoted as " $F$ " and " $M$." These need to be processed as " 0 " and " 1 " using LabelEncoder.

\section{Algorithm Used}

\section{Artificial Neural Network}

Artificial Neural Network consists of ample neurons simulated together to form a network architecture. This architecture of the neural network is used to transform the inputs into some meaningful outputs. ANN is a supervised ML technique generally used for prediction. ANN consists of an input layer, hidden layer, and output layer linked together, forming a network. These layers are associated with numerical weights to minimize the error mathematically. For the cardiac arrest prediction dataset, the input layer consists of SBP, DBP, HR, and oxygen levels, fed to 16 neurons. These input parameters are fed into the next layer. The hidden layer consists of eight neurons and finally, after the processes, gives output to the output layer as a final result.

\section{Random Forest Classifier (RFC)}

Random Forest Classifier is a type of supervised ML algorithm used for two purposes: classification and regression problems. 
TABLE 2 | Dataset description.

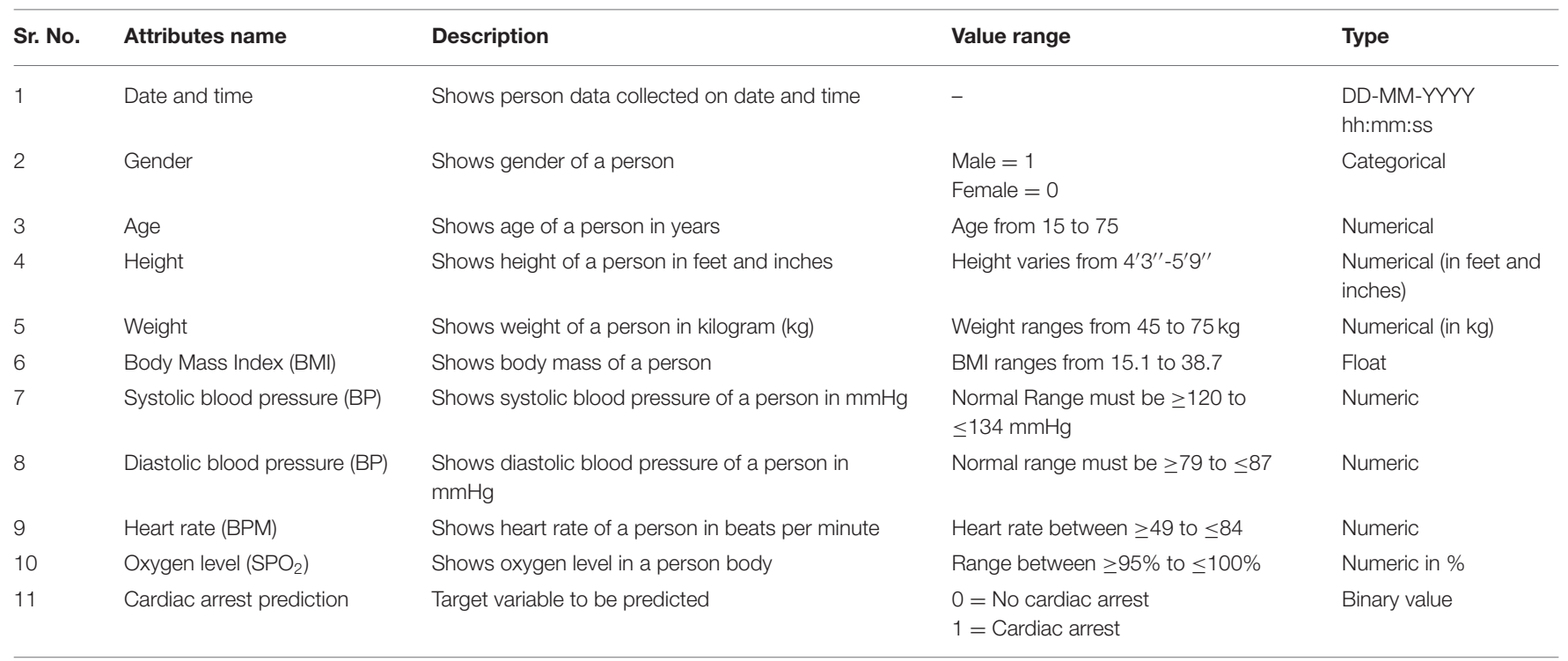

TABLE 3 | Labeled target variable.

\begin{tabular}{|c|c|c|}
\hline Parameter & "0" - predicted label & "1"-predicted label \\
\hline $\begin{array}{l}\text { Systolic blood pressure } \\
\mathrm{mmHg}\end{array}$ & $\begin{array}{l}\text { Between } \geq 120 \text { to } \\
\leq 134 \mathrm{mmHg}\end{array}$ & $\begin{array}{l}\text { Below } 120 \mathrm{mmHg} \text { and } \\
\text { above } 134 \mathrm{mmHg}\end{array}$ \\
\hline $\begin{array}{l}\text { Diastolic blood } \\
\text { pressure mmHg }\end{array}$ & $\begin{array}{l}\text { Between } \geq 79 \text { to } \leq 87 \\
\mathrm{mmHg}\end{array}$ & $\begin{array}{l}\text { Below } 79 \mathrm{mmHg} \text { and } \\
\text { above } 87 \mathrm{mmHg}\end{array}$ \\
\hline Heart rate (BPM) & Between $\geq 49$ to $\leq 84$ & Below 49 and above 84 \\
\hline Oxygen level $\left(\mathrm{SPO}_{2}\right)$ & Between 95-100\% & $\begin{array}{l}\text { Below } 95 \% \text { or above } \\
100 \%\end{array}$ \\
\hline
\end{tabular}

It follows the concept of ensemble learning, which combines multiple classifiers to solve complex problems and improves the performance of a model. Generally, it consists of several DTs forming a subset of a given dataset and improving the predictive accuracy of a dataset. RFC predicts the final output by comparing the prediction from each tree and based on the decision, such as majority voting; it indicates the result. For the cardiac arrest prediction dataset, the input parameters considered are SBP, DBP, $\mathrm{HR}$, and oxygen level. The dataset is divided into $80 \%$ training and $20 \%$ testing set and selected the number of trees estimator 30 and function "entropy" for the information gain.

\section{Gradient Boosting (XGBoost)}

"XGBoost" stands for extreme gradient boosting and can be implemented with the gradient boosted trees algorithm. XGBoost is a supervised $\mathrm{ML}$ algorithm and is considered one of the most sought-after ML techniques. This is due to its high prediction accuracy and user-friendliness. Just like other ML algorithms, XGBoost can also be used for regression and classification of problems. XGBoost uses DTs as a weak predictor and minimizes the loss function using a gradient descent algorithm. In implementation, we created an "XGB Classifier" object by passing parameters subsample $=0.7$, max_depth $=5$, and n_estimators $=100$ and by default it takes booster = "gbtree." The dataset is divided into $80 \%$ training and $20 \%$ testing set where training data are used to train a model and test part to measure its performance.

\section{Support Vector Machine}

Support Vector Machine is the best-supervised ML algorithm. It is used for both regressions and classification problems. However, for the dataset used, this algorithm is used for classification problems. For the cardiac arrest prediction dataset, the input parameters considered are SBP, DBP, HR, and oxygen level. The data are divided into $80 \%$ training and $20 \%$ testing set. We have used the Kernal value "rbf" Radial Basis Function for a non-linear classification problem.

\section{Naïve Bayes}

Naïve Bayes is a supervised ML algorithm that is used for solving a classification problem. It is generally based on the Bayes theorem and is considered one of the best classification techniques. It makes a quick prediction based on object probability. It builds a model on the classification problem and shows the high accurate result for a large dataset. For the cardiac arrest prediction dataset, the input parameters considered are SBP, DBP, HR, and oxygen level. The data are split into $80 \%$ training and $20 \%$ testing set. The model used in making a prediction is the Gaussian NB model, which follows a normal distribution for our predictor values.

\section{Decision Tree}

A DT is a classification technique used to solve a classification problem. It is a simple and widely used classification method. It is generated by providing a set of inputs in the form of a dataset. Its structure is like a flowchart that consists of Root, Sub-tree, and Leaf nodes. The algorithm starts from the root node, compares the values with the dataset attribute, and on the comparison, it follows the tree structure, i.e., branch and jumps to the next node. It continues its process until it reaches the last node, i.e., a leaf node. This helps in predicting the class of the given dataset. For the cardiac arrest prediction dataset, the input 
parameters considered are SBP, DBP, HR, and oxygen level. The data are split into $80 \%$ training and $20 \%$ testing set, considering the maximum depth of tree-level to be three and attribute used "Gini" to measure purity while creating a DT.

\section{RISK CLASSIFICATION}

Survival analysis is a statistical approach for analyzing data where the outcome variable is the time till an event occurs. They are categorized into parametric, semi-parametric, and nonparametric models. For analyzing data, several survival analysis techniques are used, such as Life Tables, Kaplan-Meier analysis, Cox proportional hazards regression model, Survival trees, and Survival random forest. In this section, the different methods we used for our survival analysis are introduced.

\section{Risk Survival Analysis Model}

In this study, two models are proposed: Age_Cox_model specific for age-based survival analysis and Gender_KM_model for gender-based survival analysis. Both models are used to find probability risk in an individual. Age_Cox_model is generally based on the Cox regression model to predict the survival probability of having different ages. Gender_KM_model is based on the Kaplan-Meier estimator to analyze the survival probability based on male and female groups.

\section{Age-Wise Classification}

The age-based classification is done using Age_Cox_model analysis. The first stage of Age_Cox_model is to provide input in the form of a dataset. The next step is the pre-process the dataset to solve the categorical data such as gender. For categorical problems, the use of LabelEncoder is applied for the gender feature. The third stage is to select relevant parts for our proposed model. After selecting features, the next step is to train our model by applying the Cox regression model. The model then predicts the result, showing the importance of features and whether there are any risks. The steps of our model are described in the following pseudocode.

Step 1: Import libraries,

Step 2: Load the dataset,

Step 3: Pre-processed the data,

Step 4: Select relevant features,

Step 5: Apply Cox regression CoxFit() model,

Step 6: Predict results show feature importance and chances of risk.

The flow of the work is presented in the below diagram, Figure 4.

The above diagram result shows the importance and risk probability of features. Table 4 describes that the $p$-value must be $<0.05$ and is considered significant.

The $p$-value of attributes age, SBP, and HR (BPM) is considered a significant covariant as values are $<0.05$.

On considering HR, i.e., exp(coef) shows the level of risk and have the following condition:

If $\mathrm{HR}=1$ Covariant has no effect of risk

$\mathrm{HR}<1$ Covariant has fewer chances of risk

HR $>1$ Covariant has more options of risk.

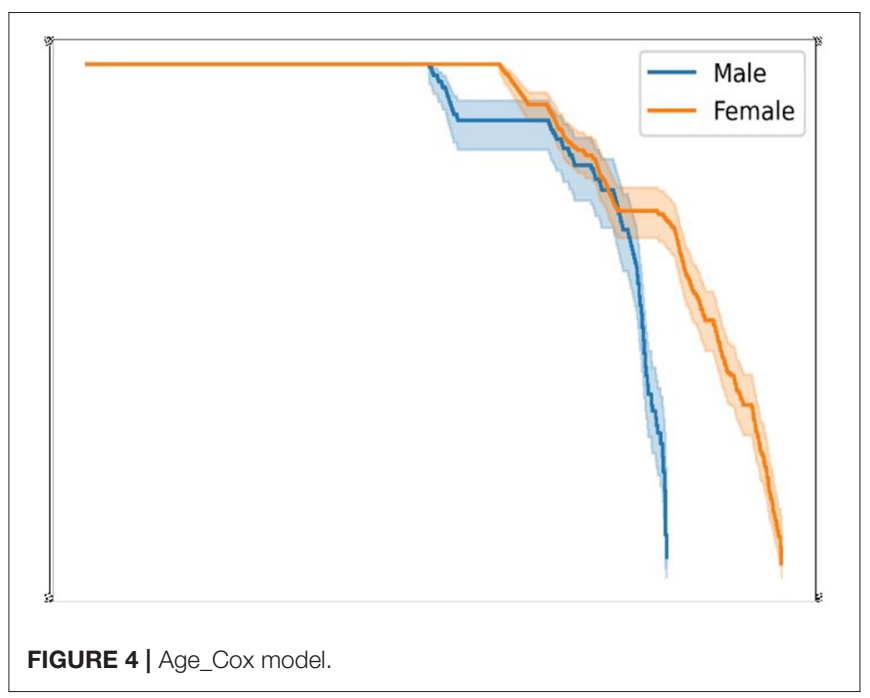

Hence, to know the chances of risk in our proposed work, age-based classification can be solved using the Cox regression survival analysis model formula, which is explained in the next part.

\section{A. Age-based risk score calculation}

The age-wise classification is derived from the prediction of a 10year risk score for cardiac arrest prediction. Among the features collected, the main risk factors for cardiac arrest prediction are a Person's age, BP (SBP and DBP), HR (measured in BPM), cardiac arrest prediction as target variable, and $\mathrm{T} 1$ factor as a time interval. The age-wise classification for 10 -year risk probability is estimated using the Cox regression model.

Table 5 represents the characteristics of risk factors that show statistical values of "total number of observations" (in each column), "number of observations with missing values," "number of observations without missing values," "minimum value" (in each variable column), "maximum value" (in each variable column), "mean value" (in each variable column), and "SD value" (in each variable column) are encapsulated.

Table 6 shows the summary statistics of events that occur while modeling risk prediction for 10 years. It shows, "total observed" $=540$ total number of data or observation, "total failed" = 328 denotes the number of possible cardiac arrests, "total censored" $=212$ denotes thin chances of cardiac arrest, and "Timestep" $=540$ denotes the number of time steps equals to the total number of observations.

The following table indicator, Table 7, represents the model quality, that is, "efficiency of the model/variable fit." The most important statistic value to be noted is the probability of the Chisquare test on the log-ratio as shown in Table 8; on comparing, the model statistics with the defined covariates, the probability value, that is, the $p$-value must be lower than $0.05(p<0.05)$, to be significant. Hence, from Table 7, we conclude that the variables show significant information.

The following table gives information on the model. Table 9 helps us understand the effect of our risk factors on the model. From Table 9, as per our collected dataset, the variable that 
TABLE 4 | Summary of Age_Cox_model.

\begin{tabular}{lrccccccccc}
\hline & $\boldsymbol{\beta}$ & $\operatorname{Exp}(\boldsymbol{\beta})$ & $\operatorname{Se}(\boldsymbol{\beta})$ & $\boldsymbol{\beta}$ lower 95\% & $\boldsymbol{\beta}$ upper 95\% & $\begin{array}{c}\operatorname{Exp}(\boldsymbol{\beta}) \\
\text { lower 95\% }\end{array}$ & $\begin{array}{c}\operatorname{Exp}(\boldsymbol{\beta}) \\
\text { upper 95\% }\end{array}$ & $\boldsymbol{z}$ & $\boldsymbol{p}$ & $-\log _{\mathbf{2}}(\boldsymbol{p})$ \\
\hline Age & 0.03 & 1.03 & 0.00 & 0.02 & 0.03 & 1.02 & 1.03 & 7.32 & $<0.005$ & 41.86 \\
Systolic BP & -0.04 & 0.96 & 0.01 & -0.05 & -0.03 & 0.95 & 0.97 & -7.35 & $<0.005$ & 42.25 \\
Diastolic BP & 0.01 & 1.01 & 0.01 & -0.01 & 0.02 & 0.99 & 1.02 & 0.72 & 0.47 & 1.09 \\
Heart rate (BPM) & 0.00 & 1.00 & 0.00 & 0.00 & 0.01 & 1.00 & 1.01 & 2.88 & $<0.005$ & 7.98 \\
\hline
\end{tabular}

TABLE 5 | Summary of statistical values of risk factors used for 10-year risk model.

\begin{tabular}{lccccc}
\hline Feature & Obs. & Min & Max & Mean & $\begin{array}{c}\text { Std. } \\
\text { deviation }\end{array}$ \\
\hline T1 & 540 & 672.00 & 1363.00 & 1076.831 & 167.757 \\
Cardiac arrest & 540 & 0.00 & 1.00 & 0.607 & 0.489 \\
prediction & & & & & \\
Age & 540 & 15.00 & 75.00 & 43.056 & 19.364 \\
Systolic BP & 540 & 82.00 & 157.00 & 118.961 & 14.896 \\
Diastolic BP & 540 & 55.00 & 125.00 & 78.113 & 9.905 \\
Heart rate (BPM) & 540 & 24.00 & 238.00 & 87.006 & 39.929 \\
\hline
\end{tabular}

TABLE 6 | Summary statistics (events).

\begin{tabular}{lccc}
\hline $\begin{array}{l}\text { Total } \\
\text { observed }\end{array}$ & $\begin{array}{c}\text { Total } \\
\text { failed }\end{array}$ & $\begin{array}{c}\text { Total } \\
\text { censored }\end{array}$ & $\begin{array}{c}\text { Time } \\
\text { steps }\end{array}$ \\
\hline 540 & 328 & 212 & 540 \\
\hline
\end{tabular}

TABLE 7 | The goodness of fit statistic.

\begin{tabular}{lcc}
\hline Statistic & Independent & Full \\
\hline Observations & 328.000 & 328.000 \\
DF & 0.000 & 4.000 \\
-2 Log (likelihood) & 3487.800 & 3398.055 \\
AIC & 3487.800 & 3406.055 \\
SBC & 3487.800 & 3421.227 \\
Iterations & 1.000 & 3.000
\end{tabular}

TABLE 8 | Test of the null hypothesis $\mathrm{HO}$ : beta $=0$.

\begin{tabular}{llll}
\hline Statistic & DF & Chi-square & Pr $>\mathbf{C h i}^{\mathbf{2}}$ \\
\hline -2 Log (likelihood) & 4 & 89.74558 & $<0.0001$ \\
Score & 4 & 94.97911 & $<0.0001$ \\
Wald & 4 & 94.87288 & $<0.0001$ \\
\hline
\end{tabular}

influences our risk survival model is SBP. From the beginning of the study, this risk variable indicates the patient has a high effect on cardiac arrest prediction. In this study, from the table, the "Value" column shows the HR, which is obtained as the estimated parameter exponent and is calculated using Equation (1).
The HR is given using the mentioned formula:

$$
\mathrm{H}(\mathrm{t})=\mathrm{h}_{0}(\mathrm{t}) \exp \left(\sum \beta_{\mathrm{i}} \mathrm{X}_{\mathrm{i}}\right)(\mathrm{i}=1 \text { to } \mathrm{r})(1)
$$

Notations are:

$\mathrm{t}=$ Survival time (10-year risk) $(\mathrm{t}=10)$.

$\exp =$ Exponential function $(\exp (\mathrm{X}))$.

$B_{i}=$ It measures the impact of covariates $\left(B_{1}, B_{2}, B_{3}, \ldots, B_{r}\right)$.

$\mathrm{X}_{\mathrm{i}}=$ Predictor variables $\left(\mathrm{X}_{1}, \mathrm{X}_{2}, \mathrm{X}_{3}, \ldots, \mathrm{X}_{\mathrm{r}}\right)$.

$\mathrm{h}_{0}(\mathrm{t})=$ Baseline hazard rate.

$\mathrm{H}(\mathrm{t})=\mathrm{HR}$.

The Cox model regression has an exponential form, as seen in Equation (1).

As per our dataset, for 10 years, the term $\mathrm{h}_{0}(\mathrm{t})=$ baseline survival rate value is considered 0.993 at mean values of covariates from the survival distribution function. Here, " $t$ " represents an event that occurred at a time. The $\mathrm{H}(\mathrm{t})$ hazard function is determined by the set of covariates $\mathrm{X}_{\mathrm{i}}=\left(\mathrm{X}_{1}, \mathrm{X}_{2}, \mathrm{X}_{3}, \ldots, \mathrm{X}_{\mathrm{r}}\right)$ and the regression coefficient $B_{i=}\left(B_{1}, B_{2}, B_{3}, \ldots, B_{r}\right)$ that measures the impact of covariates.

In Table 10, the column "rho," correlates between residuals and time vector as in our above case-Kaplan Meier. The other two columns, "Chi-square" and "Pr > Chi-square," show test statistics and the associated $p$-value. Here, the $p$-values must be $<0.005$, and the value from Table 10 reveals no violation of the risk assumption.

Hence, to find the survival risk score from the Cox model regression, it can be written as:

$$
\mathrm{R}(\mathrm{t})=1-\mathrm{R} 0(\mathrm{t}) \exp \left(\sum \beta \mathrm{iXi}-\sum \beta \mathrm{i} \overline{\mathrm{X}} \mathrm{i}\right)(\mathrm{i}=1 \text { to } \mathrm{r})
$$

$\mathrm{R}(\mathrm{t})$ is the risk estimation of cardiac arrest of an individual; $\mathrm{R}_{0}(\mathrm{t})$ is the baseline survival time at $\mathrm{t} ; \beta i$ is the regression coefficient covariates values mentioned in Table 8; $\mathrm{X}_{\mathrm{i}}$ is the value of the $i_{\text {th }}$ : the risk factor value [log-transformed value natural $\log (\ln )]$; and $\bar{X}_{I}$ is the mean value of the covariates. Here, the $\mathrm{i}$ value ranges from 1 to $\mathrm{r}$ and denotes the risk factors considered. The cardiac arrest risk score or percent can be determined using the mentioned formula as shown in Equation (2).

The estimation of the Cox regression model is formulated from the scoresheet, and the Cox regression equation is derived. Tables 11, 12 show the scoresheet that represents the points allocated 
TABLE 9 | Regression coefficients.

\begin{tabular}{|c|c|c|c|c|c|c|c|}
\hline Variable & Value & Std. error & Wald Chi-square & $\mathrm{Pr}>\mathrm{Chi}^{2}$ & HR & HR $<95 \%$ & HR > 95\% \\
\hline Age & 0.027 & 0.004 & 53.587 & $<0.0001$ & 1.027 & 1.020 & 1.035 \\
\hline Systolic BP & -0.039 & 0.005 & 54.068 & $<0.0001$ & 0.962 & 0.000 & 0.972 \\
\hline Diastolic BP & 0.005 & 0.007 & 0.517 & 0.472 & 1.005 & 0.991 & 1.019 \\
\hline Heart rate (BPM) & 0.004 & 0.001 & 8.232 & 0.004 & 1.004 & 1.001 & 1.007 \\
\hline
\end{tabular}

TABLE 10 | Proportionality test.

\begin{tabular}{lccc}
\hline Variable & Rho & Chi-square & Pr $>\mathbf{C h}^{\mathbf{2}}$ \\
\hline Age & -0.22834 & 24.24338 & $<0.0001$ \\
Systolic BP & 0.09274 & 4.133863 & 0.042 \\
Diastolic BP & 0.012658 & 0.061134 & 0.805 \\
Heart rate (BPM) & 0.136702 & 7.842881 & 0.005 \\
Global & & 32.0064 & $<0.0001$ \\
\hline
\end{tabular}

TABLE 11 | Cardiac arrest risk score for men.

\begin{tabular}{lccc}
\hline \multicolumn{2}{c}{ Male } \\
\hline Points & Age & Blood pressure & Heart rate \\
\hline-1 & $30-34$ & $<130 / 85$ & $<50$ \\
0 & $35-39$ & $130 / 85-139 / 89$ & $50-70$ \\
1 & $40-44$ & $140 / 90-159 / 99$ & $70-90$ \\
2 & $45-49$ & $\geq 160 / 100$ & \\
3 & $50-54$ & & \\
4 & $55-59$ & & \\
5 & $60-64$ & & \\
6 & $65-69$ & & \\
7 & $70-74$ & & \\
\hline
\end{tabular}

TABLE 12 | Cardiac arrest risk score for women.

\begin{tabular}{lccc}
\hline \multicolumn{2}{c}{ Female } & \\
\hline Points & Age & Blood pressure & Heart rate \\
\hline-9 & $30-34$ & & $<50$ \\
-4 & $35-39$ & $<120 / 89$ & $50-70$ \\
0 & $40-44$ & $120 / 80-139 / 89$ & $70-90$ \\
3 & $45-49$ & $140 / 90-159 / 99$ & $90+$ \\
6 & $50-54$ & $\geq 160 / 100$ & \\
7 & $55-59$ & & \\
\hline
\end{tabular}

to an individual risk factor for both genders. Table 13 shows the total risk score probability after formulating Equation (2).

The calculation for estimation of cardiac arrest risk is derived below:
TABLE 13 | Ten-year risk for the Risk points calculated.

\begin{tabular}{lccc}
\hline & \multicolumn{2}{c}{$\mathbf{1 0}$-year risk score (\%) } & \\
\hline Points & Male & Points & Female \\
\hline-3 & $1 \%$ & $<-2$ & $1 \%$ \\
-2 or -1 & $2 \%$ & $-1,0$, or 1 & $2 \%$ \\
0 & $3 \%$ & 2 or 3 & $3 \%$ \\
1 or 2 & $4 \%$ & 4 & $4 \%$ \\
3 & $6 \%$ & 5 & $5 \%$ \\
4 & $7 \%$ & $6 \%$ & $6 \%$ \\
5 & $9 \%$ & 7 & $7 \%$ \\
6 & $11 \%$ & 8 & $8 \%$ \\
7 & $14 \%$ & 9 & $9 \%$ \\
8 & $18 \%$ & 10 & $11 \%$ \\
9 & $22 \%$ & 11 & $13 \%$ \\
10 & $27 \%$ & 12 & $15 \%$ \\
11 & $33 \%$ & 13 & $17 \%$ \\
12 & $40 \%$ & 14 & $20 \%$ \\
13 & $47 \%$ & 15 & $24 \%$ \\
14 or more & $56 \%$ & 16 & $27 \%$ \\
& & 17 or more & $32 \%$ \\
\hline
\end{tabular}

TABLE 14 | Risk point calculation.

\begin{tabular}{lcc}
\hline Risk factor involved & Values & Points allocated \\
\hline Gender & Female & - \\
Age & 50 & 6 \\
Systolic blood pressure & 131 & 0 \\
Diastolic blood pressure & 80 & 0 \\
Heart rate & 112 & 3 \\
Total points scored & & 9 \\
Risk estimation according to sheet & & $9 \%$ \\
\hline
\end{tabular}

\section{B. Risk estimation from the scoresheet}

As mentioned in Tables 11, 12, the risk points are calculated according to the covariate's ranges. Table 14 shows points allocated according to the risk factor involved.

\section{Risk estimation from Cox regression model}

As per Equation (2), risk estimation of cardiac arrest in female is given by:

Formula:

$$
\mathrm{R}(\mathrm{t})=1-\mathrm{R} 0(\mathrm{t}) \exp \left(\sum \beta \mathrm{BXi}-\sum \beta \mathrm{X} \overline{\mathrm{X}} \mathrm{i}\right)(\mathrm{i}=1 \text { to } \mathrm{r})
$$


Values for:

- $\mathrm{R}_{0}(\mathrm{t})$ for $\mathrm{t}=10$ years $=0.993$ is the baseline survival

- $\beta i$ is the estimated regression coefficient (in Table 9)

- $X i$ is the log-transformed value of ith risk factor

- $\bar{X} I$ is the mean value of the covariates considered (in Table 5)

- $\mathrm{R}$ is the number of risk factors involved in predicting risk. The risk estimation of the cardiac arrest based on the Cox regression model is computed as:

- $X i$ is the log-transformed value of ith risk factor

- $\bar{X} I$ is the mean value of the covariates considered (in Table 5)

- $\mathrm{R}$ is the number of risk factors involved in predicting risk.

The risk estimation of the cardiac arrest based on the Cox regression model is computed as:

$$
\begin{aligned}
\sum_{i=1}^{r} \beta i X i & =\left[0.027^{*} \ln (50)\right]+\left[-0.039^{*} \ln (131)\right] \\
& +\left[0.005^{*} \ln (80)\right]+\left[0.004^{*} \ln (112)\right] \\
& =0.1056--0.1901+0.0219+0.0188 \\
& =-0.0438 \\
\sum_{{ }_{i=1}^{r} \beta i \bar{X} i} & =\left[0.027^{*} 43.056\right]+\left[-0.039^{*} 118.961\right] \\
& +\left[0.005^{*} 78.113\right]+\left[0.004^{*} 87.006\right] \\
& =1.1625-4.6394+0.3905+0.3480 \\
& =-2.7384
\end{aligned}
$$

Substituting values in Equation (2) we get,

$$
\begin{aligned}
R(t) & =1-R_{0}(t) \exp \left(\sum_{i=1}^{r} \beta i X i-\sum_{i=1}^{r} \beta i \bar{X} i\right) \\
& =1-\mathrm{R}_{0}(10) \exp (-0.0438-(-2.7384)) \\
& =1-(0.993) \exp (2.6946) \\
& =1-(0.993)(14.79) \\
& =1-0.9013 \\
& =0.0987 \\
& =0.0987^{*} 100(\text { convertingto } \%) \\
& =9.87 \\
& \approx 9 \%
\end{aligned}
$$

According to the scoresheet, the point system is $9 \%$, and the Cox regression model also gives the approximate percentage of risk $9 \%$. Therefore, a female having age 50 may have a chance of $9 \%$ more risk of cardiac arrest in the coming 10-year risk prediction.

\section{Gender-Wise Classification}

The gender-based classification is done using Gender_KM_model analysis. The first stage of Gender_KM_model is to provide input in the form of a dataset. The next step is to pre-process the dataset to solve the categorical data such as gender. For categorical problems, the use of LabelEncoder is applied for the gender feature. We divide our dataset into two groups: male and female and apply the Kaplan-Meier analysis model. The model then predicts the result, showing prediction probability in each gender at an event time. The steps of our model are described in the following pseudocode.

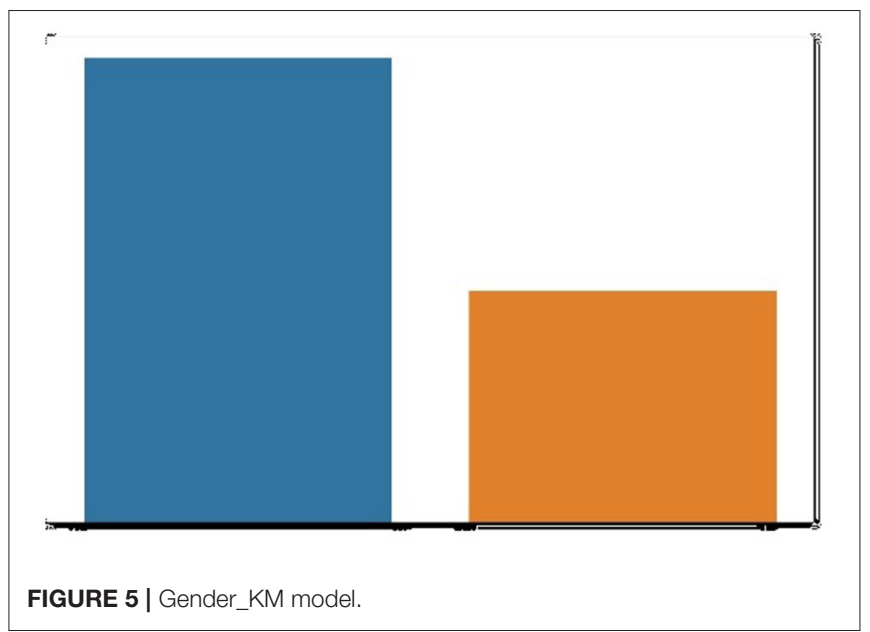

Step 1: Import libraries

Step 2: Load the dataset

Step 3: Pre-processed the data

Step 4: Divide dataset into two groups: male and female

Step 5: Apply Kaplan-Meier KaplanMeierFitter() model

Step 6: Predict results shows probability in each gender at an event time.

The flow of the work is presented in the below diagram, Figure 5.

\section{A. Gender-based estimation of risk probability}

The gender-wise risk classification can be estimated using a nonparametric survival estimator called the Kaplan-Meier method. It is useful in determining the survival probabilities of an individual and gender-wise. As per our collected dataset, survival function estimator $\mathrm{S}(\mathrm{t})$ can be given as:

$$
\mathrm{S}(\mathrm{t})=\Pi(1-\mathrm{di} / \mathrm{ni})(\mathrm{i}: \mathrm{ti}<=\mathrm{t})
$$

Where,

$\mathrm{S}(\mathrm{t})=$ gives survival probability

$t_{i}=$ time at when an event occurs

$\mathrm{d}_{\mathrm{i}}=$ number of events occur (e.g., at risk of cardiac arrest) at time ti

$\mathrm{n}_{\mathrm{i}}=$ number of individuals who have survived (e.g., not at risk of cardiac arrest) at time $t_{i}$

The Kaplan-Meier estimator helps to find the probability or survival rates of adjusted covariates. In our dataset, our estimation is to classify risk according to gender (male and female). We have divided our data into two groups: male and female. Our target is to notice any significant difference in probability or survival rate if data are divided into two groups based on gender. Based on gender split, we come up with survival probability in men and women based on Equation (3), which is shown in Tables 15, 16.

The survival probability/risk of an individual (male/female) is estimated based on the time interval. On predicting survival probabilities in both genders using object creation of KaplanMeier we get, 
TABLE 15 | Event table for male.

\begin{tabular}{|c|c|c|c|c|c|}
\hline Event_at & Removed & Observed $\left(d_{i}\right)$ & Censored & Entrance & At_risk $\left(\mathrm{n}_{\mathrm{i}}\right)$ \\
\hline 0 & 0 & 0 & 0 & 180 & 180 \\
\hline 672 & 1 & 1 & 0 & 0 & 180 \\
\hline 674 & 1 & 0 & 1 & 0 & 179 \\
\hline 676 & 1 & 1 & 0 & 0 & 178 \\
\hline 678 & 1 & 1 & 0 & 0 & 177 \\
\hline 680 & 1 & 1 & 0 & 0 & 176 \\
\hline 682 & 1 & 0 & 1 & 0 & 175 \\
\hline 684 & 1 & 0 & 1 & 0 & 174 \\
\hline 686 & 1 & 0 & 1 & 0 & 173 \\
\hline 688 & 1 & 1 & 0 & 0 & 172 \\
\hline 690 & 1 & 1 & 0 & 0 & 171 \\
\hline 692 & 1 & 0 & 1 & 0 & 170 \\
\hline 694 & 1 & 0 & 1 & 0 & 169 \\
\hline 696 & 1 & 1 & 0 & 0 & 168 \\
\hline 698 & 1 & 1 & 0 & 0 & 167 \\
\hline$\cdots \cdots$ & $\cdots \cdots$ & $\cdots \cdots$ & $\cdots \cdots$ & $\ldots \ldots$ & $\ldots \ldots$ \\
\hline 1,120 & 2 & 1 & 1 & 0 & 20 \\
\hline 1,122 & 2 & 0 & 2 & 0 & 18 \\
\hline 1,124 & 2 & 0 & 2 & 0 & 16 \\
\hline 1,126 & 2 & 1 & 1 & 0 & 14 \\
\hline 1,128 & 2 & 0 & 2 & 0 & 12 \\
\hline 1,130 & 2 & 1 & 1 & 0 & 10 \\
\hline 1,132 & 2 & 1 & 1 & 0 & 8 \\
\hline 1,134 & 2 & 1 & 1 & 0 & 6 \\
\hline 1,136 & 2 & 2 & 0 & 0 & 4 \\
\hline 1,138 & 2 & 1 & 1 & 0 & 2 \\
\hline
\end{tabular}

kmf_m.predict $(900)=0.89061 \ldots \approx 89 \%$

kmf_f.predict $(900)=0.92205 \ldots \approx 92 \%$

Here, predict (900) indicates predicting survival or risk probability at 900-time intervals. The male probability of surviving is $89 \%$, and the female probability of surviving is $92 \%$.

From the above observation, we can conclude that the survival probability of women is higher compared to men. Men are at higher risk than women. Below, Figure 6 shows the survival probability of men and women at a given time $t$.

\section{EXPLORATORY DATA ANALYSIS}

Figure 7 shows the number of female and male data in our dataset from the figure. We can conclude that there are 150 "men" and 350 "women."

Figure 8 shows a correlation graph among attributes, using the syntax:

\section{data.corr( )}

Figure 9 shows counts of cardiac arrest prediction " 1 " and " 0 " in each gender, male and female. We conclude that 100 "men" are prone to cardiac arrest, and around 75 "men" are risk free of cardiac arrest. Similarly, more than 200 "women" are prone to cardiac arrest, and $\sim 140$ "women" are risk free from cardiac arrest.
TABLE 16 | Event table for female.

\begin{tabular}{|c|c|c|c|c|c|}
\hline Event_at & Removed & Observed $\left(d_{i}\right)$ & Censored & Entrance & At_risk $\left(\mathrm{n}_{\mathrm{i}}\right)$ \\
\hline 0 & 0 & 0 & 0 & 360 & 360 \\
\hline 810 & 1 & 1 & 0 & 0 & 360 \\
\hline 812 & 1 & 1 & 0 & 0 & 359 \\
\hline 814 & 1 & 1 & 0 & 0 & 358 \\
\hline 816 & 1 & 1 & 0 & 0 & 357 \\
\hline 818 & 1 & 1 & 0 & 0 & 356 \\
\hline 820 & 1 & 1 & 0 & 0 & 355 \\
\hline 822 & 1 & 1 & 0 & 0 & 354 \\
\hline 824 & 1 & 0 & 1 & 0 & 353 \\
\hline 826 & 1 & 1 & 0 & 0 & 352 \\
\hline 828 & 1 & 1 & 0 & 0 & 351 \\
\hline 830 & 1 & 1 & 0 & 0 & 350 \\
\hline 832 & 1 & 1 & 0 & 0 & 349 \\
\hline$\cdots \cdots$ & $\ldots \ldots$ & $\ldots \ldots$ & $\ldots \ldots$ & $\ldots \ldots$ & $\ldots \ldots$ \\
\hline 1,345 & 2 & 1 & 1 & 0 & 20 \\
\hline 1,347 & 2 & 2 & 0 & 0 & 18 \\
\hline 1,349 & 2 & 1 & 1 & 0 & 16 \\
\hline 1,351 & 2 & 1 & 1 & 0 & 14 \\
\hline 1,353 & 2 & 1 & 1 & 0 & 12 \\
\hline 1,355 & 2 & 1 & 1 & 0 & 10 \\
\hline 1,357 & 2 & 1 & 1 & 0 & 8 \\
\hline 1,359 & 2 & 0 & 2 & 0 & 6 \\
\hline 1,361 & 2 & 1 & 1 & 0 & 4 \\
\hline 1,363 & 2 & 1 & 1 & 0 & 2 \\
\hline
\end{tabular}

Figure 10 shows the various age distributions. It is observed from the bar plot that 25 years of people are seen more following 17 and 63 years of people are found more.

Figure 11 shows the distribution of age, and it seems to be normally distributed.

\section{IMPLEMENTATION DETAILS}

\section{Experimental Setup}

In this research, we implemented six supervised ML algorithms to evaluate the possibility of cardiac arrest and the performance of a classifier in a classification problem. For evaluation, the real-time dataset was collected using sensors and equipment as described in section Methodology. The dataset consists of 10 attributes, among which five main attributes, i.e., the vital signs of the patient, are mainly considered. For experimental execution, the "Data Streamer" tool was used in this study to collect the pulse rate every $2 \mathrm{~min}$. Data Streamer is an open-source tool used to collect live data supporting capturing, analyzing, and visualizing real-time sensor data in Excel.

As described in section Methodology, the next step uses an ML model: ANN, RFC, XGBoost, SVM, NB, and DT classifier. Before applying the model to our collected data, the collected dataset was imported using "read_csv()," and then the data was pre-processed as discussed in section Methodology. In the next step, we did feature selection using "StandardScaler()" where the vital signs of a patient were majorly considered. Furthermore, the dataset was 


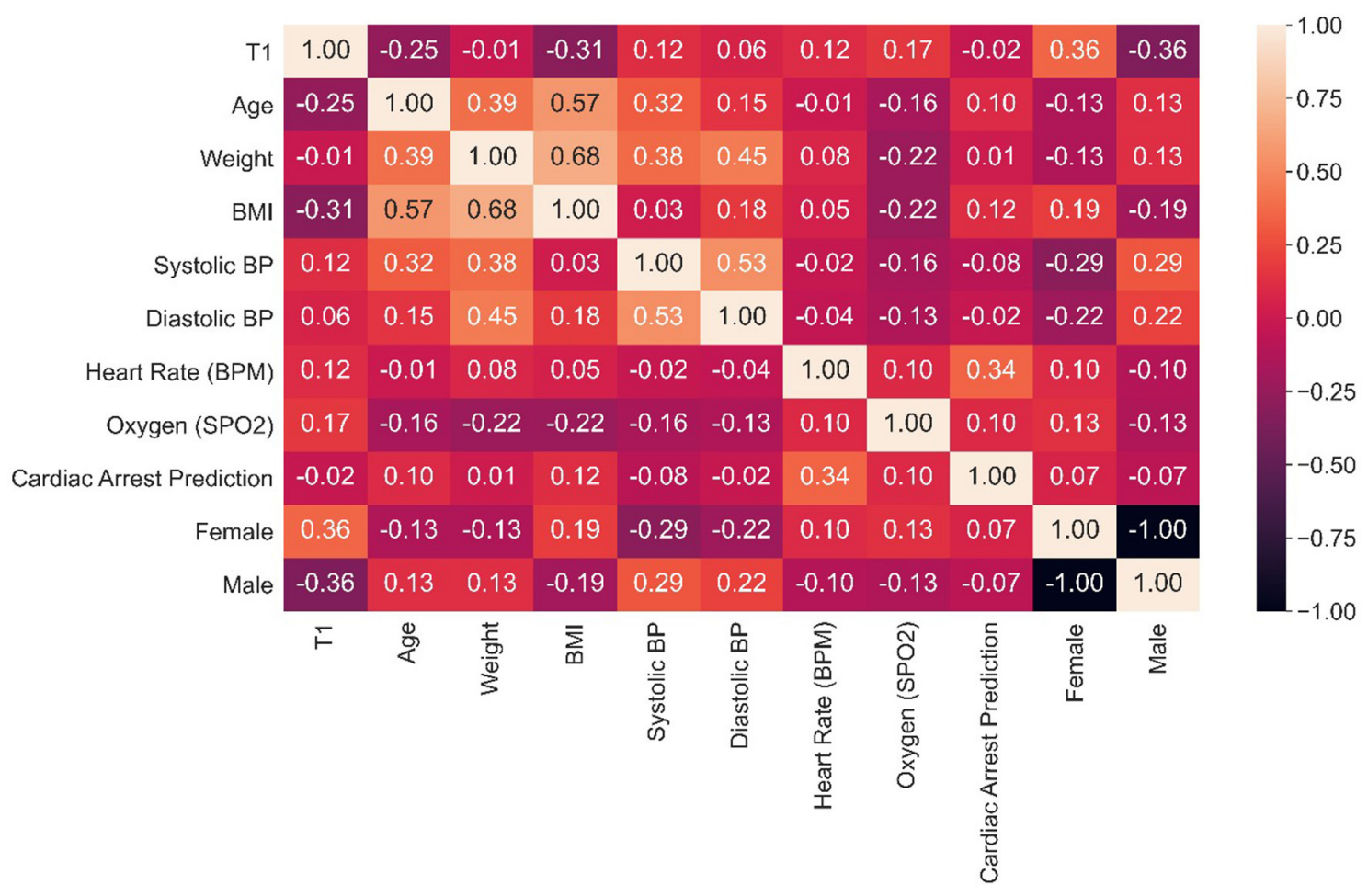

FIGURE 6 | Men/women survival probability graph.

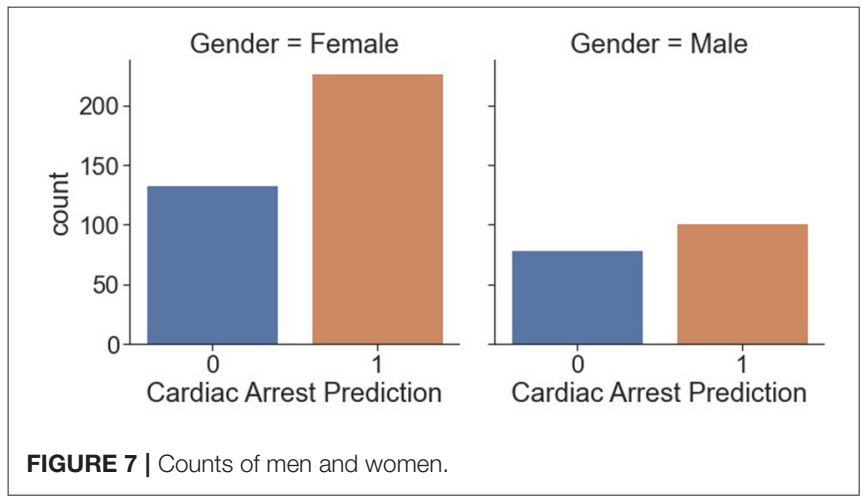

split up into $80 \%$ training and $20 \%$ testing set. Then, models were applied to these data to check model accuracy. In the next phase, to test the performance of the classifier, various experimental metrics were computed. The contribution part of our research involves gender-wise analysis of risk/survival probability using the Kaplan-Meier algorithm, and age-wise risk probability for 10 years was formulated using the "XLSTAT" add-in tool in Excel. XLSTAT is an open-source software used to analyze, customize, and display results in Excel. Hence, the experiments discussed above have been carried out using Anaconda software in a Jupyter notebook python environment using ML libraries on an Intel(R) Core (TM) i5-3320M CPU @ 2.60 GHz system.

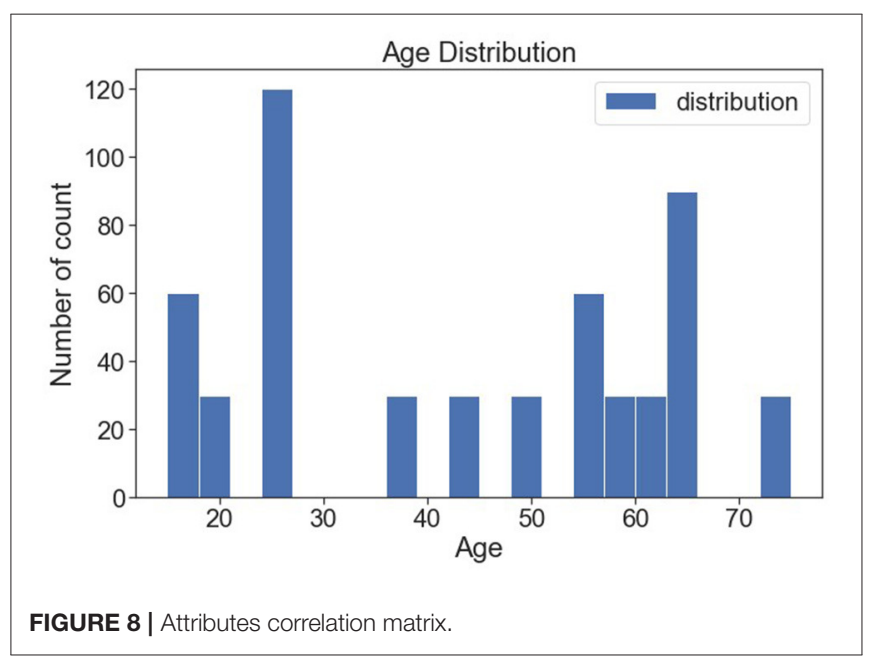

\section{Evaluation Metrics}

The evaluation metrics of the model are formulated with the confusion matrix. For this, the calculation of values is measured based on:

- True positive (TP) $=$ number of occurrences that are correctly determined.

- False negative $(\mathrm{FN})=$ number of occurrences that are incorrectly predicted and not required. 


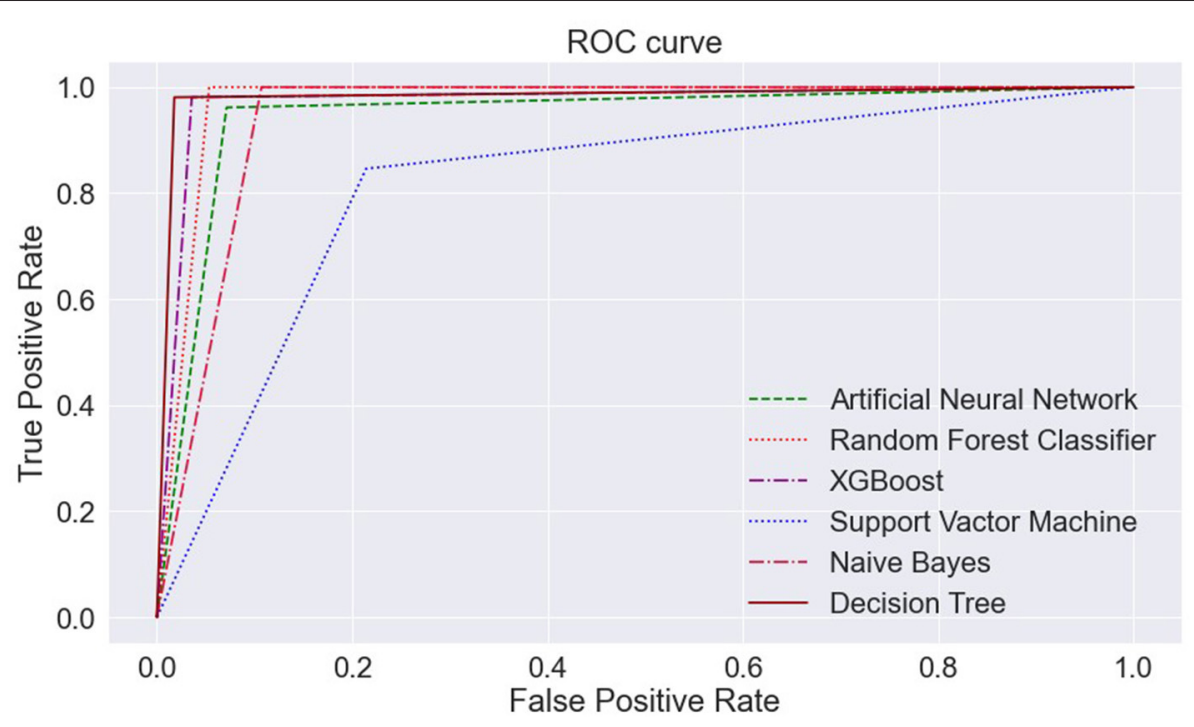

FIGURE 9 | Counts of cardiac arrest in men/women.

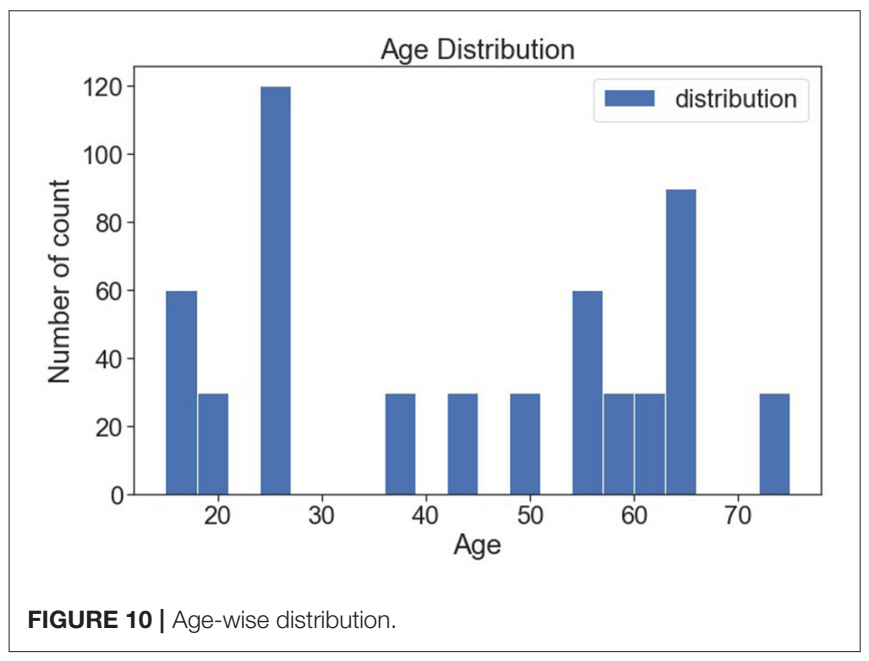

- False positive $(\mathrm{FP})=$ number of occurrences that are incorrectly predicted.

- True negative $(\mathrm{TN})=$ number of occurrences that are correctly predicted and not required.

Based on this parameter, we have calculated four measurements with the given formula:

- Accuracy $=T N+T P / T N+T P+F N+F P$

- Precision $=T P / T P+F P$

- Sensitivity $($ Recall $)=T P / T P+F N$

- F1 Score $=2^{*}\left(\right.$ Precision $^{*}$ Recall $/$ Precision + Recall $)$

These measurements help to know the cardiac risk associated with heart diseases.

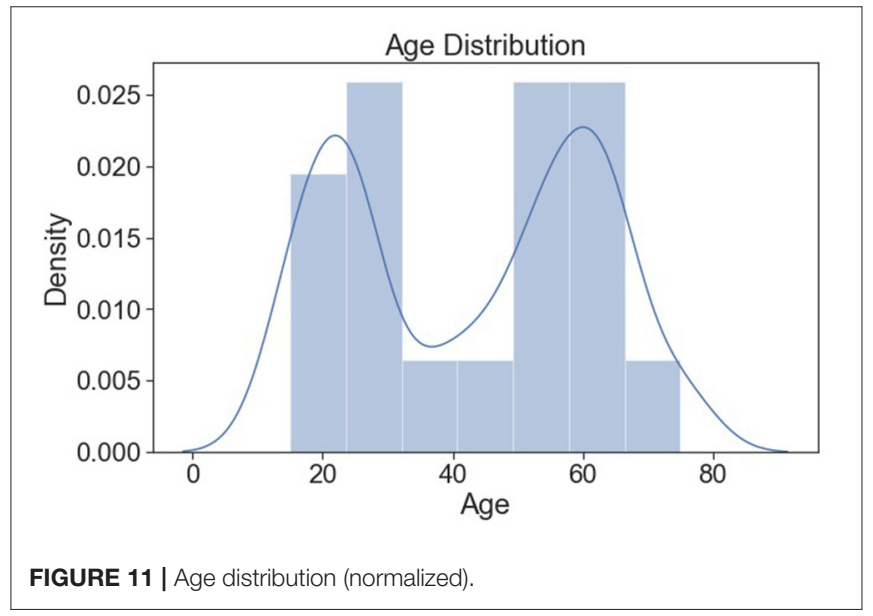

\section{Results}

After executing the ML model on the collected dataset, we found the accuracy, sensitivity, precision, and F1-score of each algorithm, as shown in Table 14. These measurements were calculated with the support of confusion matrix value TP, TN, FP, and FN for each algorithm shown in Figures 12A-F.

Table 17 shows the Precision, Recall, F1- score, and overall accuracy of ML algorithms. The value for Precision, Recall, and F1- score is calculated against 2-the class problem of having cardiac arrest risk and no cardiac arrest risk. The label "0" has been used for no risk in cardiac diseases, and " 1 " has been used for risk in cardiac disease.

Hence, Table 17 concludes that the DT outperforms all ML algorithms achieving overall $96 \%$ accuracy.

Figures 13A-F portrays the area under the curve (AUC) and receiver operating characteristic (ROC) curve. ROC curve depicts 

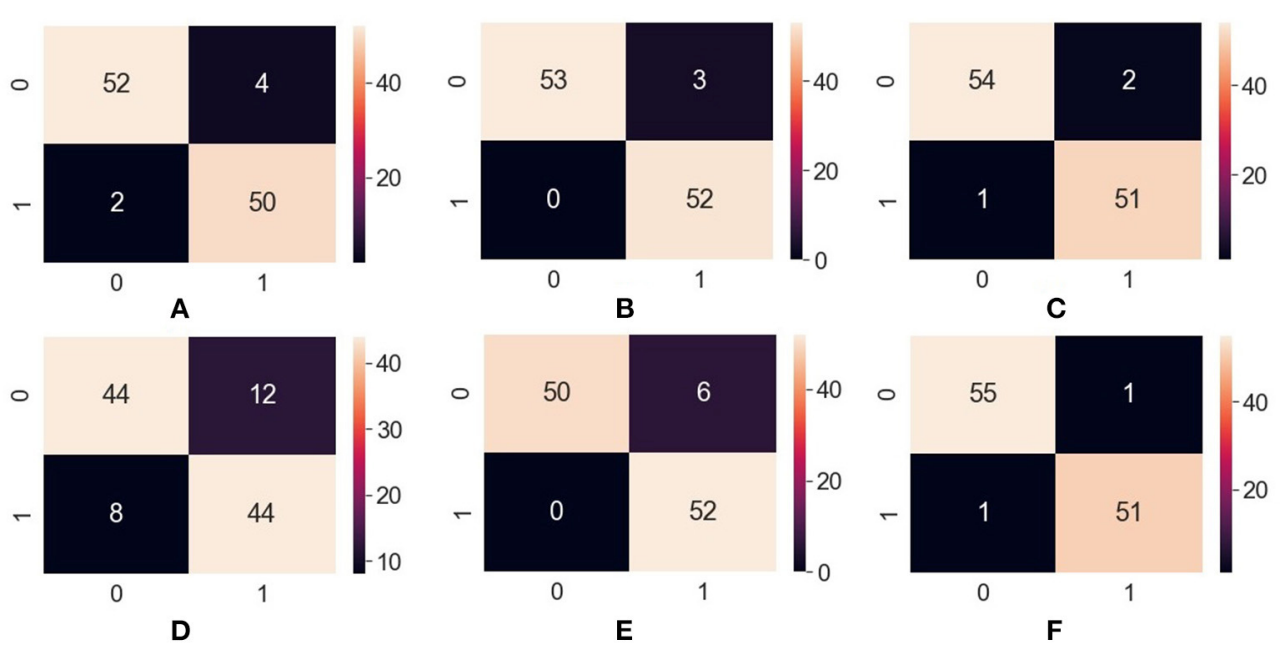

FIGURE 12 | Confusion matrix-(A) ANN, (B) RFC, (C) XGBoost, (D) SVM, (E) Naïve Bayes, (F) Decision Tree. ANN, Artificial Neural Network; RFC, Random Forest Classifier; XGBoost, Extreme Gradient Boosting; SVM, Support Vector Machine.

TABLE 17 | Model performance.

\begin{tabular}{|c|c|c|c|c|c|}
\hline Algorithm & Overall accuracy & Label & Precision & Recall & F1-score \\
\hline \multirow[t]{2}{*}{ Decision tree } & 0.98 & 0 & 0.98 & 0.98 & 0.98 \\
\hline & & 1 & 0.98 & 0.98 & 0.98 \\
\hline \multirow[t]{2}{*}{ Gradient boosting } & 0.97 & 0 & 0.94 & 0.98 & 0.97 \\
\hline & & 1 & 0.98 & 0.96 & 0.97 \\
\hline \multirow[t]{2}{*}{ Random forest classifier } & 0.97 & 0 & 0.94 & 1.00 & 0.97 \\
\hline & & 1 & 1.00 & 0.94 & 0.97 \\
\hline \multirow[t]{2}{*}{ Artificial neural network } & 0.94 & 0 & 0.92 & 0.96 & 0.94 \\
\hline & & 1 & 0.96 & 0.92 & 0.94 \\
\hline \multirow[t]{2}{*}{ Support vector machine } & 0.81 & 0 & 0.78 & 0.84 & 0.81 \\
\hline & & 1 & 0.84 & 0.78 & 0.81 \\
\hline \multirow[t]{2}{*}{ Naïve bayes } & 0.94 & 0 & 0.89 & 1.00 & 0.94 \\
\hline & & 1 & 1.00 & 0.89 & 0.94 \\
\hline
\end{tabular}

the graphical appearance to measure the model performance. It shows the true negative, and true positive values plotted at distinct levels of the threshold.

The AUC curve represents the aggregate measures of performance at different threshold values. It also describes the probability value from the classification model to get an accurate result. If the AUC curve value shows 0 value, it predicts wrong results, and if 1 , it means an exact model. Here, in Figure 14, the accuracy of all three classification models reaches 1 value, which means our prediction accuracy is highly accurate.

\section{DISCUSSION, CONCLUSION, AND FUTURE WORK}

\section{Discussion}

This study is aimed to detect and predict whether the patient is prone to cardiac arrest or not. This research was carried out using six ML classification algorithms, namely, ANN, RFC, XGBoost, SVM, NB, and DT classifier on a real-time collected dataset. Different tools were conducted for analyzing and classifying data on Intel(R) Core (TM) i5-3320M CPU @ $2.60 \mathrm{GHz}$ system.

Dataset was split up into $0.8 \%$ training and $0.2 \%$ testing set, and then data pre-processing was done. To get the result, ML algorithms were applied to datasets to get the accuracy of the result. The results-accuracy, Precision, Recall, and F1-score-were generated using Python programming on Jupyter notebook.

Figures 15A,B show the scores. Precision, Recall, and F1scores of the patients having no risk or chances of cardiac arrest and patients with a risk of cardiac arrest. These scores help us to understand the measure of relevance of accuracy.

Comparing both charts, we conclude that "DT" reaches the maximum accuracy based on the scores retrieved from Precision, Recall, and F1 scores. 

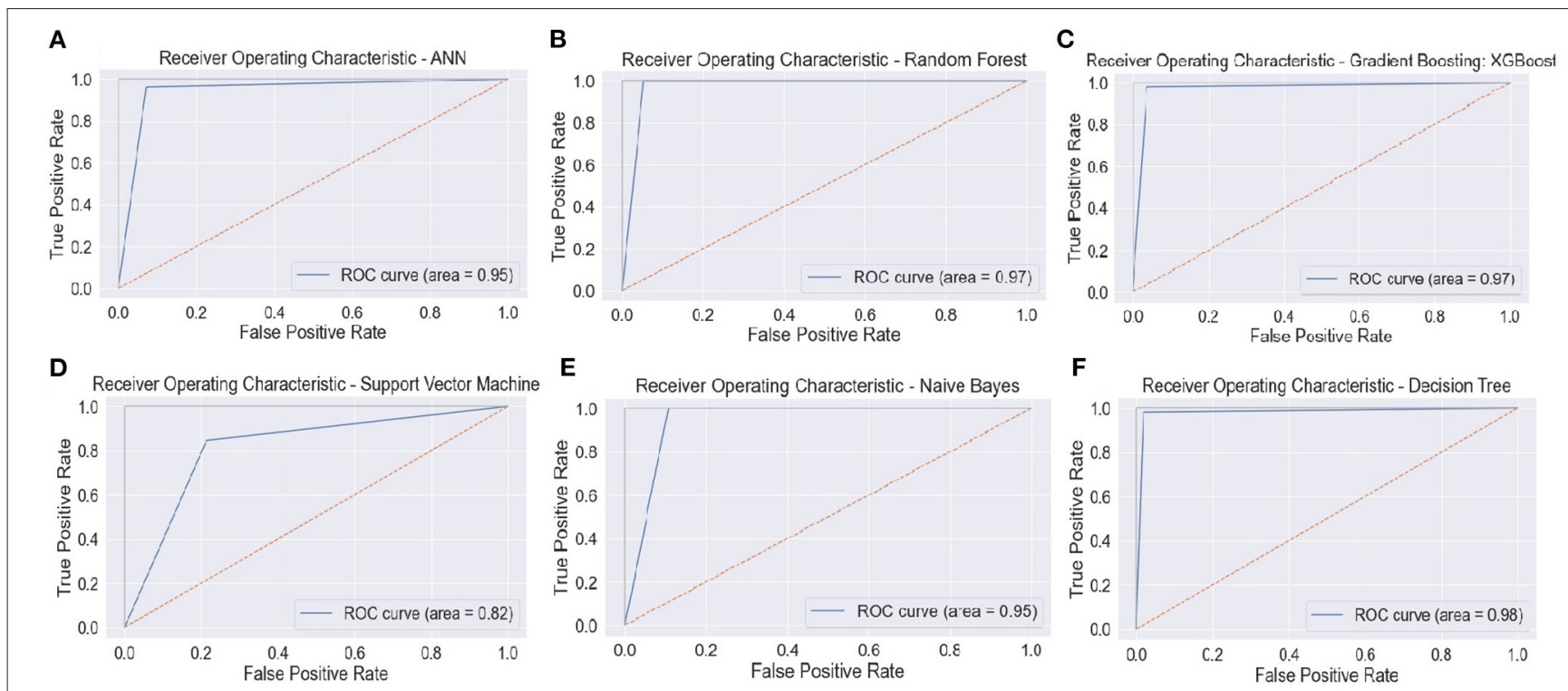

FIGURE 13 | ROC curve-(A) ANN, (B) RFC, (C) XGBoost, (D) SVM, (E) Naïve Bayes, and (F) Decision Tree. ANN, Artificial Neural Network; RFC, Random Forest Classifier; XGBoost, Extreme Gradient Boosting; SVM, Support Vector Machine.

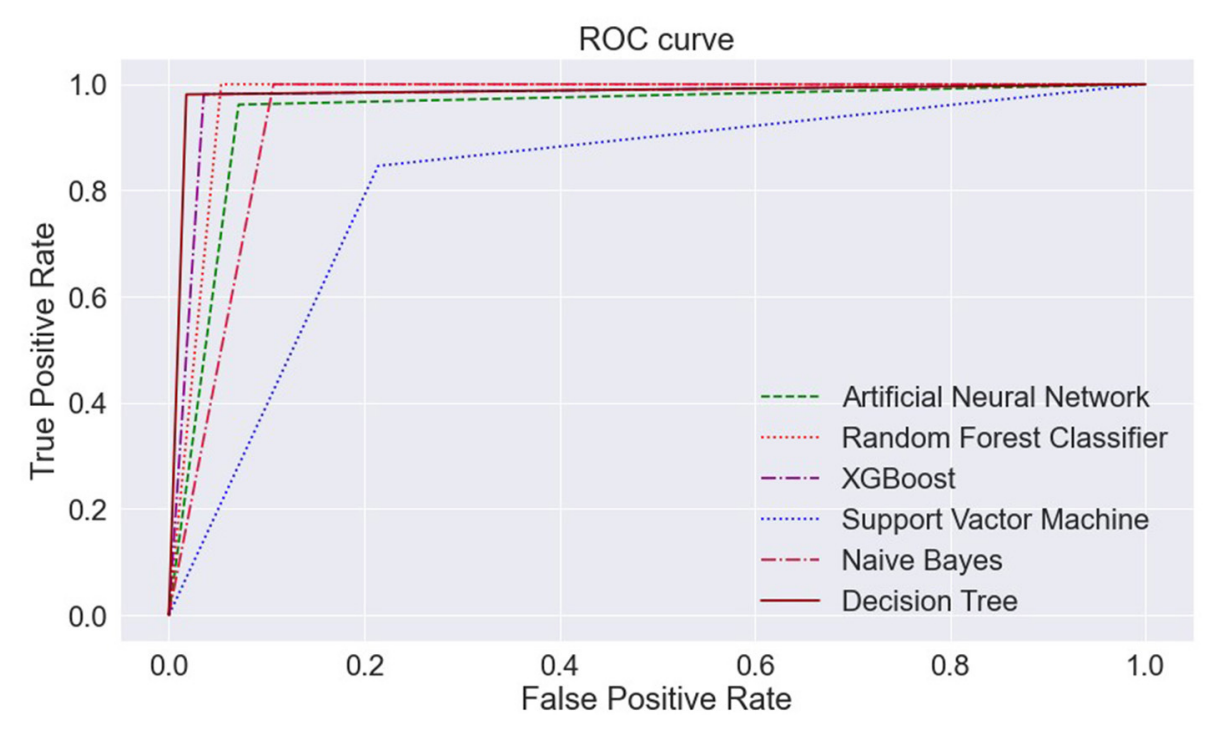

FIGURE 14 | ML models-ROC curve. ML, machine learning; ROC, receiver operating characteristic.

Furthermore, the main contribution of our research work shows an effective and efficient method for the prediction of cardiac arrest risk in gender-wise and different age-wise people in terms of survival probability. The method Kaplan-Meier shows statistical analysis in gender for showing the chances of survival probability. The result obtained by applying the KaplanMeier model to our collected dataset shows that female survival probability is more than that in men, as shown in Figure 6. Another method, Cox regression analysis, is another statistical analysis method that shows prediction/survival probability for the next 10 years of risk in cardiac arrest for different age groups. We can use this model to see cardiac risk in an individual. In addition, this method helps identify whether a person is at risk in the coming next 10 years using the score sheet displayed above.

\section{Conclusion}

This study contributes classification techniques for detecting and comparing gender-based and age-based probability for cardiac arrest survival. The main objective of our work is to 


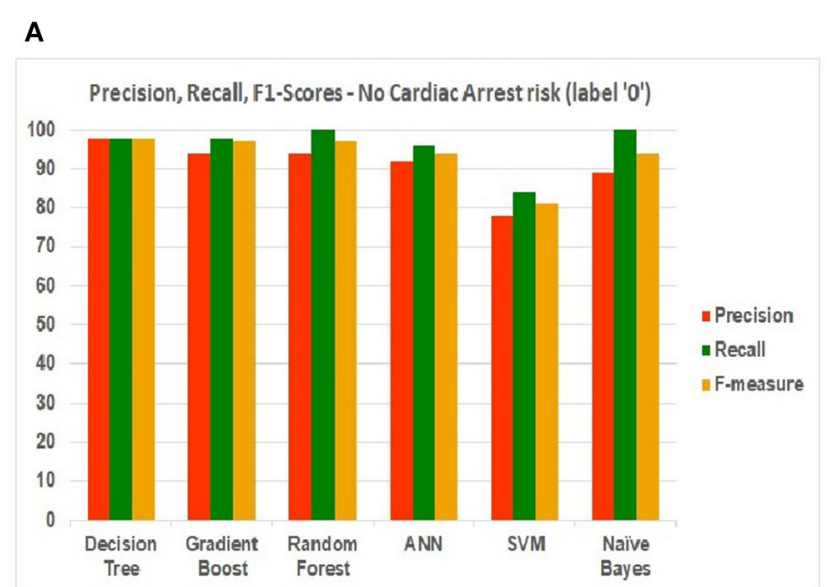

B

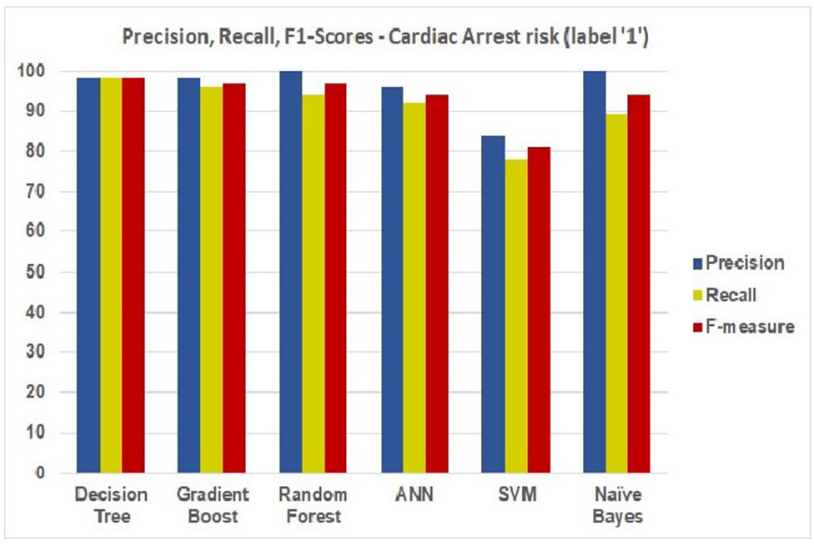

FIGURE 15 | ML models_Precision, Recall, and F1-scores-for (A) label "0" (B) label "1."

recognize cardiac arrest in a patient as early as possible using an ML model. Apart from that, our main contribution shows survival probability in an individual with gender-based and age-based using two effective methods: Kaplan-Meier and Cox regression methods. From the result, in detecting cardiac arrest risk in gender-based survival probability, female patients of our collected record show higher chances of survival than male patients. While in detecting cardiac arrest risk in age-based survival probability, patients with age 30 and more may have chances of cardiac risk as per our proposed model. From our classification model, the predicting results of the DT outperform the other ML classifiers.

\section{Future Study}

This study can be further extended by adding more diseases and making predictions using different classifier models. In addition

\section{REFERENCES}

1. Ambekar S, Phalnikar R. Disease risk prediction by using convolutional neural network. In: Fourth International Conference on Computing Communication Control and Automation (ICCUBEA). Pune (2018). p. 1-5. doi: 10.1109/ICCUBEA.2018.8697423

2. Ramprakash P, Sarumathi R, Mowriya R, Nithyavishnupriya S. Heart disease prediction using deep neural network. In: 2020 International Conference on Inventive Computation Technologies (ICICT). Coimbatore (2020). p. 66670. doi: 10.1109/ICICT48043.2020.9112443

3. Shah D, Patel S, Bharti SK. Heart disease prediction using machine learning techniques. SN Comput Sci. (2020) 1:345. doi: 10.1007/s42979-020-00365-y

4. Deepa N, Prabadevi B, Maddikunta PK, Gadekallu TR, Baker T, Khan $\mathrm{MA}$, et al. An AI-based intelligent system for healthcare analysis using Ridge-Adaline Stochastic Gradient Descent Classifier. J Supercomput. (2021) 77:1998-2017. doi: 10.1007/s11227-020-03347-2

5. Maini E, Venkateswarlu B, Gupta A. Applying machine learning algorithms to develop a universal cardiovascular disease prediction system. In: International Conference on Intelligent Data Communication Technologies and Internet of Things. (2018). p. 627-632. doi: 10.1007/978-3-030-03146-6_69 to that, we can add more features, and predictions can be made on a larger dataset.

\section{DATA AVAILABILITY STATEMENT}

The raw data supporting the conclusions of this article will be made available by the authors, without undue reservation.

\section{AUTHOR CONTRIBUTIONS}

SP and SA: conceptualization and design. SP, SA, and AS: data collection, manuscript writing, conceptualization, and design. SP, $\mathrm{SA}, \mathrm{AS}$, and KK: manuscript proofreading, manuscript writing, conceptualization, and design. SR: revisions, conceptualization, manuscript writing, and manuscript proofreading. All authors contributed to the article and approved the submitted version.

6. Mohan S, Thirumalai C, Srivastava G. Effective heart disease prediction using hybrid machine learning techniques. IEEE Access. (2019) 7:8154254. doi: 10.1109/ACCESS.2019.2923707

7. Fitriyani NL, Syafrudin M, Alfian G, Rhee J. HDPM: an effective heart disease prediction model for a clinical decision support system. IEEE Access. (2020) 8:133034-50. doi: 10.1109/ACCESS.2020.3010511

8. Sarmah SS. An efficient IoT-based patient monitoring and heart disease prediction system using deep learning modified neural network. IEEE Access. (2020) 8:135784-97. doi: 10.1109/ACCESS.2020.3007561

9. Srinivasu PN, Bhoi AK, Jhaveri RH, Reddy GT, Bilal M. Probabilistic deep Q network for real-time path planning in censorious robotic procedures using force sensors. J Real Time Image Proc. (2021) 18:14359. doi: 10.1007/s11554-021-01122-x

10. Chauhan U, Kumar V, Chauhan V, Tiwary S, Kumar A. Cardiac arrest prediction using machine learning algorithms. In: 2019 2nd International Conference on Intelligent Computing, Instrumentation and Control Technologies (ICICICT). (2019). p. 886-90. doi: 10.1109/ICICICT46008.2019.8993296

11. Reddy GT, Reddy MP, Lakshmanna K, Rajput DS, Kaluri R, Srivastava G. Hybrid genetic algorithm and a fuzzy logic classifier for heart 
disease diagnosis. Evol Intell. (2020) 13:185-96. doi: 10.1007/s12065-01900327-1

12. Singh A, Kumar R. Heart disease prediction using machine learning algorithms. In: 2020 International Conference on Electrical and Electronics Engineering (ICE3). (2020). p. 452-7. doi: 10.1109/ICE348803.2020.91 22958

13. Li JP, Haq AU, Din SU, Khan J, Khan A, Saboor A. Heart disease identification method using machine learning classification in e-healthcare. IEEE Access. (2020) 8:107562-82. doi: 10.1109/ACCESS.2020.3001149

14. Pan Y, Fu M, Cheng B, Tao X, Guo J. Enhanced deep learning assisted convolutional neural network for heart disease prediction on the internet of medical things platform. IEEE Access. (2020) 8:18950312. doi: 10.1109/ACCESS.2020.3026214

15. Garg A, Sharma B, Khan R. Heart disease prediction using machine learning techniques. IOP Conf Ser. (2021) 1022:012046. doi: 10.1088/1757-899X/1022/1/012046

16. Yadav KK, Sharma A, Badholia A. Heart disease prediction using machine learning techniques. Inform Technol Industry. (2021) 9:20714. doi: 10.17762/itii.v9i1.120

17. Latha CB, Jeeva SC. Improving the accuracy of prediction of heart disease risk based on ensemble classification techniques. Inform Med Unlocked. (2019) 16:100203. doi: 10.1016/j.imu.2019.100203

18. Singh $H$, Navaneeth NV, Pillai GN. Multisurface proximal SVM based decision trees for heart disease classification. In: TENCON 2019-2019 IEEE Region 10 Conference (TENCON). (2019). p. 13-18. doi: 10.1109/TENCON.2019.8929618

19. Sowmiya C, Sumitra P. A hybrid approach for mortality prediction for heart patients using ACO-HKNN. J Ambient Intell Hum Comput. (2021) 12:5405-12. doi: 10.1007/s12652-020-02027-6

20. Alotaibi FS. Implementation of machine learning model to predict heart failure disease. Int J Adv Comput Sci Appl. (2019) 10:2618. doi: 10.14569/IJACSA.2019.0100637

21. Nikookar E, Naderi E. Hybrid ensemble framework for heart disease detection and prediction. Int J Adv Comput Sci Appl. (2018) 9:18. doi: 10.14569/IJACSA.2018.090533

22. Tama BA, Im S, Lee S. Improving an intelligent detection system for coronary heart disease using a two-tier classifier ensemble. BioMed Res Int. (2020) 2020:9816142. doi: 10.1155/2020/9816142

23. Tate AR, Rao GH. Activity trackers, wearables, noninvasive technologies for early detection, and management of cardiometabolic risks. Int J Biomed. (2020) 189-97. doi: 10.21103/Article10(3)_RA2

24. Prabhu T, Darshana J, Dharani Kumar M, Hansaa Nazreen M. Health risk prediction by machine learning over data analytics. Int Res J Eng Technol. (2019) 6:606-11. doi: 10.21203/rs.2.24678/v1

25. Shankar V, Kumar V, Devagade U, Karanth V, Rohitaksha K. Heart disease prediction using CNN algorithm. SN Comput Sci. (2020) 1:170. doi: 10.1007/s42979-020-0097-6

26. Amin P, Anikireddypally NR, Khurana S, Vadakkemadathil S, Wu W. Personalized health monitoring using predictive analytics. In" 2019 IEEE Fifth International Conference on Big Data Computing Service and Applications (BigDataService). Las Vegas, NV (2019). p. 271-8. doi: 10.1109/BigDataService.2019.00048
27. Chen Y, Zhang L, Qin X, Yi B. Preoperative risk prediction of heart failure with numerical and textual attributes. Int J Innov Comput Inform Control. (2020) 16:2035-46. doi: 10.24507/ijicic.16.06.2035

28. Cohen S, Liu A, Wang F, Guo L, Brophy JM, Abrahamowicz M, et al. Risk prediction models for heart failure admissions in adults with congenital heart disease. Int J Cardiol. (2021) 322:149-57. doi: 10.1016/j.ijcard.2020. 08.039

29. Chang D, Chang D, Pourhomayoun M. Risk prediction of critical vital signs for icu patients using recurrent neural network. In: 2019 International Conference on Computational Science and Computational Intelligence (CSCI). (2019). p. 1003-6. doi: 10.1109/CSCI49370.2019. 00191

30. Youssef Ali Amer A, Wouters F, Vranken J, de Korte-de Boer D, Smit-Fun V, Duflot $P$, et al. Vital signs prediction and early warning score calculation based on continuous monitoring of hospitalised patients using wearable technology. Sensors. (2020) 2020:6593. doi: 10.3390/s202 26593

31. Stehlik J, Schmalfuss C, Bozkurt B, Nativi-Nicolau J, Wohlfahrt P, Wegerich S, et al. Continuous wearable monitoring analytics predict heart failure hospitalization: the LINK-HF multicenter study. Circulation. (2020) 13:e006513. doi: 10.1161/CIRCHEARTFAILURE.119.006513

32. Jia X, Baig MM, Mirza F, GholamHosseini H. A cox-based risk prediction model for early detection of cardiovascular disease: identification of key risk factors for the development of a 10-Year CVD risk prediction. Adv Prevent Med. (2019) 2019:8392348. doi: 10.1155/2019/83 92348

33. Yang L, Wu H, Jin X, Zheng P, Hu S, Xu X, et al. Study of cardiovascular disease prediction model based on random forest in eastern China. Sci Rep. (2020) 10:5245. doi: 10.1038/s41598-020-62133-5

34. Made F, Nonterah EA, Tlotleng N, Ntlebi V, Naicker N. Ten-year risk of fatal cardiovascular disease and its association with metabolic risk factors among waste pickers in South Africa. BMC Cardiovasc Disord. (2021) 21:336. doi: $10.21203 /$ rs.3.rs-236769/v1

Conflict of Interest: The authors declare that the research was conducted in the absence of any commercial or financial relationships that could be construed as a potential conflict of interest.

Publisher's Note: All claims expressed in this article are solely those of the authors and do not necessarily represent those of their affiliated organizations, or those of the publisher, the editors and the reviewers. Any product that may be evaluated in this article, or claim that may be made by its manufacturer, is not guaranteed or endorsed by the publisher.

Copyright (C) 2021 Shah, Ahirrao, Pandya, Kotecha and Rathod. This is an openaccess article distributed under the terms of the Creative Commons Attribution License (CC BY). The use, distribution or reproduction in other forums is permitted, provided the original author(s) and the copyright owner(s) are credited and that the original publication in this journal is cited, in accordance with accepted academic practice. No use, distribution or reproduction is permitted which does not comply with these terms. 